\title{
In Vivo Calcium Imaging Reveals Functional Rewiring of Single Somatosensory Neurons after Stroke
}

\author{
Ian R. Winship ${ }^{1,2}$ and Timothy H. Murphy ${ }^{1}$ \\ ${ }^{1}$ Department of Psychiatry, Brain Research Centre, University of British Columbia, Vancouver, British Columbia, Canada V6T 1Z3, and ${ }^{2}$ Neurochemical \\ Research Unit, Department of Psychiatry and Centre for Neuroscience, University of Alberta, Edmonton, Alberta, Canada T6G 2G3
}

Functional mapping and microstimulation studies suggest that recovery after stroke damage can be attributed to surviving brain regions taking on the functional roles of lost tissues. Although this model is well supported by data, it is not clear how activity in single neurons is altered in relation to cortical functional maps. It is conceivable that individual surviving neurons could adopt new roles at the expense of their usual function. Alternatively, neurons that contribute to recovery may take on multiple functions and exhibit a wider repertoire of neuronal processing. In vivo two-photon calcium imaging was used in adult mice within reorganized forelimb and hindlimb somatosensory functional maps to determine how the response properties of individual neurons and glia were altered during recovery from ischemic damage over a period of 2-8 weeks. Single-cell calcium imaging revealed that the limb selectivity of individual neurons was altered during recovery from ischemia, such that neurons normally selective for a single contralateral limb processed information from multiple limbs. Altered limb selectivity was most prominent in border regions between stroke-altered forelimb and hindlimb macroscopic map representations, and peaked 1 month after the targeted insult. Two months after stroke, individual neurons near the center of reorganized functional areas became more selective for a preferred limb. These previously unreported forms of plasticity indicate that in adult animals, seemingly hardwired cortical neurons first adopt wider functional roles as they develop strategies to compensate for loss of specific sensory modalities after forms of brain damage such as stroke.

Key words: stroke; ischemia; two-photon; calcium; intrinsic optical signals; forelimb; hindlimb; plasticity; functional imaging; remapping

\section{Introduction}

Recovery of function after stroke-induced cortical injury is thought to occur through adaptive plasticity in surviving neurons that replaces the function of lost networks. Functional imaging of stroke patients (Chollet et al., 1991; Ward et al., 2003a,b, 2006; Fridman et al., 2004; Jaillard et al., 2005; Rossini et al., 2007) and intracranial microstimulation (ICMS) studies in animal models (Castro-Alamancos and Borrel, 1995; Nudo and Milliken, 1996; Friel et al., 2000; Frost et al., 2003; Kleim et al., 2003; Gharbawie et al., 2005) suggest that surviving cortex can adopt the motor or sensory processing functions of regions lost to damage. Functional imaging and ICMS are powerful tools for assessing regional plasticity, but lack the spatial and temporal resolution to define

\footnotetext{
Received Feb. 11, 2008; revised April 24, 2008; accepted May 22, 2008.

This work was supported by a Canadian Institutes of Health Research (CIHR) operating grant to T.H.M. (MOP49586) and a Focus on Stroke postdoctoral Fellowship to I.R.W. (jointly funded by the Heart and Stroke Foundation of Canada, CIHR, Canadian Stroke Network, and AstraZeneca Canada). Partial support for surgery, hardware, and software development was derived from the University of British Columbia In Vivo Imaging Centre (funded by CIHR Grant PRG-80144). We thank Ping Li, Pumin Wang, and Heidi Erb for excellent assistance with surgical preparations and mouse colonies, Craig Brown and Kathryn Todd for comments on this manuscript, Jamie Boyd and Kerry Delaney for development of imaging software and hardware, and Alexander Goroshkov for technica assistance with the two-photon microscope.

Correspondence should be addressed to Dr. Timothy H. Murphy, Department of Psychiatry, Brain Research Centre, 4N1-2255 Wesbrook Mall, University of British Columbia, Vancouver, British Columbia, Canada V6T 1Z3. E-mail: thmurphy@interchange.ubc.ca.

I. R. Winship's present address: Neurochemical Research Unit, Department of Psychiatry and Centre for Neuroscience, University of Alberta, Edmonton, Alberta, Canada T6G 2 G3.

D0I:10.1523/JNEUROSCI.0622-08.2008

Copyright $\odot 2008$ Society for Neuroscience $\quad$ 0270-6474/08/286592-15\$15.00/0
}

how activity in single neurons or local neuronal ensembles are changing relative to infarct boundaries. Few studies have examined the poststroke stimulus-evoked activity of individual neurons and local networks in vivo (Reinecke et al., 2003), and none have assessed the properties of individual neurons and glia with high resolution relative to reorganized sensory maps. Conceivably, changes to single neurons may not mirror aggregate circuit behavior, and insight into mechanisms of circuit recovery can be made by studying sensory responses of individual neurons after stroke. Analysis of individual neurons can help determine whether stroke-induced plasticity is achieved by surviving neurons giving up their usual function or whether individual neurons may be able to process multiple sensory streams.

Regarding the spatial aspects of stroke-induced rewiring, studies of brain structure indicate heightened plasticity in periinfarct cortex, with enhanced axonal sprouting, synaptogenesis, and turnover of dendritic spines (Stroemer et al., 1995; Xerri et al., 1998; Cramer and Chopp, 2000; Biernaskie and Corbett, 2001; Carmichael et al., 2001; Carmichael, 2003, 2006; Gonzalez and Kolb, 2003; Brown et al., 2007). Interestingly, dendritic spine plasticity is greatest within $<1 \mathrm{~mm}$ of the infarct (Brown et al., 2007), indicating the need to evaluate both the function and spatial location of individual neurons in close proximity to the periinfarct cortex. Here, we use regional imaging of intrinsic optical signals (IOSs) to define macroscopic remapping in somatosensory cortex of mice with targeted ischemic insults (Zhang et al., 2005; Schaffer et al., 2006; Brown et al., 2007; Zhang and Murphy, 

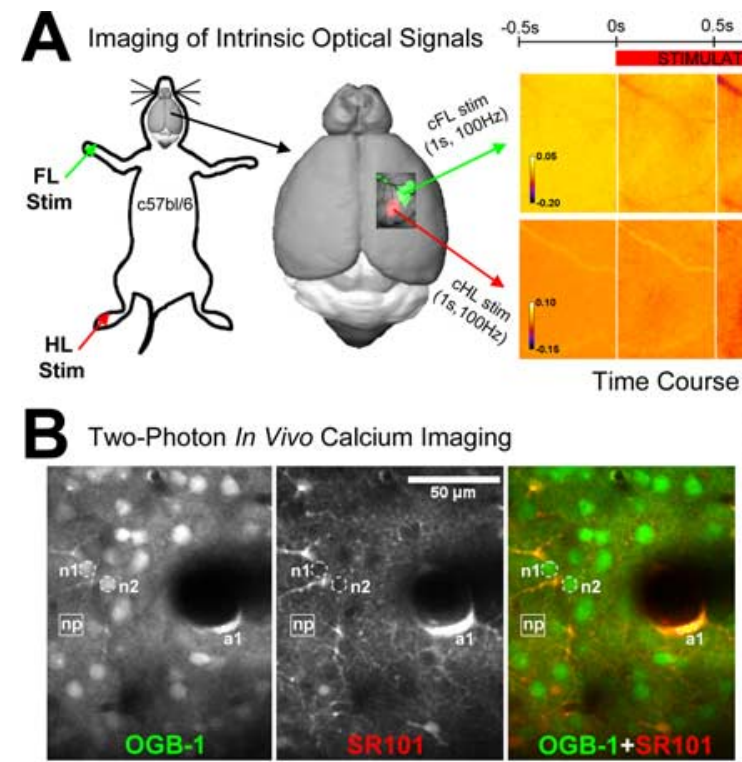

$\frac{0.5 \mathrm{~s}}{1.5}$ $1.0 \mathrm{~s} \quad 1.5 \mathrm{~s}$

B

Two-Photon In Vivo Calcium Imaging
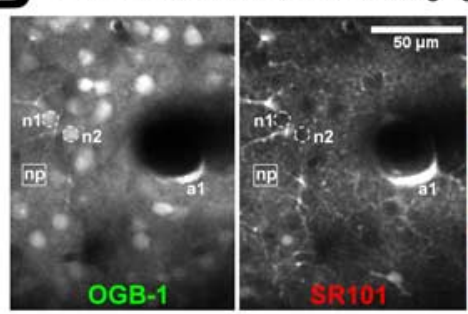

C

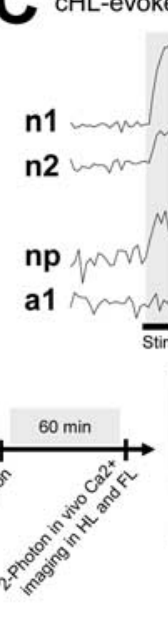

D Experimental Timeline
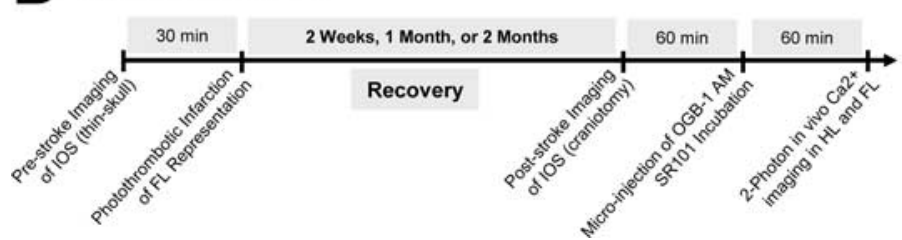

Figure 1. Imaging protocol and experimental timeline. $\boldsymbol{A}$, Vibrotactile stimulation $(1 \mathrm{~s}, 100 \mathrm{~Hz})$ of the $\mathrm{CFL}$ or CHL during imaging of IOSs was used to define S1FL and S1HL, respectively. The time course and regional distribution of the IOS response to cFL (top) and cHL (bottom) stimulation are shown in $\boldsymbol{A}$. Each frame in the time course shows the percentage change in light intensity across the IOS image over a 0.5 s period before, during, or after limb stimulation. 105 response maps (see Materials and Methods) were thresholded at $50 \%$ of peak response amplitude and merged with an image of the surface vasculature to create color-coded regional maps of cFL and CHL activation, as shown in $\boldsymbol{A}$ (far right). The membrane-permeant $\mathrm{Ca}^{2+}$ indicator $0 \mathrm{~GB}-1$ AM and astrocyte marker SR101 were applied to facilitate two-photon $\mathrm{Ca}^{2+}$ imaging within areas defined by 105 imaging. $\boldsymbol{B}$, Two-photon images, acquired in vivo, of OGB-1 and SR101 labeling in S1HL in the region demarcated by the dashed black box in the thresholded map in $\boldsymbol{A}$ (depth, $147 \mu \mathrm{m}$ ). $\mathrm{Ca}^{2+}$ imaging in both S1FL and S1HL during vibrotactile stimulation was used to assess sensory-evoked single-cell responses. $C$, Mean $\mathrm{Ca}^{2+}$ signals, averaged from 10 trials, from the neurons ( $\mathrm{n} 1$ and $\mathrm{n2}$ ), astrocyte (a1), and region of the neuropil (np) denoted in $\boldsymbol{B}$. CHL stimulation evoked strong $\mathrm{Ca}^{2+}$ transients in $\mathrm{n1}$, $\mathrm{n} 2$, and the neuropil, but not in a1. Imaging, targeted stroke, and recovery were performed according to the experimental timeline shown in $\boldsymbol{D}$.

2007). We then perform in vivo two-photon calcium imaging at different sites within these regional maps, as previously used in the visual and somatosensory cortex of unlesioned animals (Stosiek et al., 2003; Ohki et al., 2005, 2006; Kerr et al., 2007; Mrsic-Flogel et al., 2007; Sato et al., 2007; Winship et al., 2007). By optically recording neuronal activity using in vivo $\mathrm{Ca}^{2+}$ imaging, we assessed stroke-induced changes in sensory-evoked function and spatial organization of $>10,000$ individual, visually identified cells in cortical layers $2 / 3$. We provide evidence for greatly enhanced receptive fields in peri-infarct neurons (and in particular those at map boundaries), such that 1 month after stroke, previously limb-selective neurons and glia process information from multiple limbs. With longer recovery, these cells developed more specific response characteristics and exhibited a defined limb preference.

\section{Materials and Methods}

Data acquired from C57BL/6 mice (male, $2-4$ months of age; $n=34$ ) are included in this report. Only those mice in which both regional and two-photon imaging data were successfully acquired are included in this dataset. Mice were maintained on a $12 \mathrm{~h}$ light/dark cycle and were given standard laboratory diet and water ad libitum. The experimental timeline is shown in Figure $1 D$. All experiments were conducted in accordance $1.5 \mathrm{~s}$
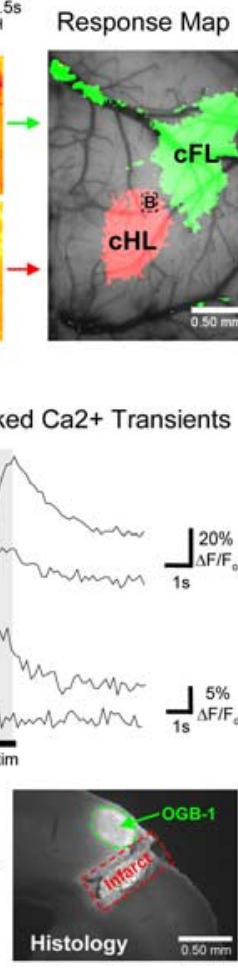

with the guidelines set forth by the Canadian Council for Animal Care.

Identification of forelimb somatosensory cortex. Mice were anesthetized with isoflurane (2\% induction, $1.5 \%$ maintenance, in $100 \%$ air) and placed in a stereotaxic frame. Body temperature was maintained at $37^{\circ} \mathrm{C}$ using a rectal probe coupled to a temperature regulator and heating pad. A midline incision of the scalp was made to expose the skull overlying primary forelimb somatosensory cortex (S1FL). In some mice $(n=$ 12), S1FL was located based on stereotaxic coordinates $(\sim 1.5-3 \mathrm{~mm}$ lateral; -0.5 to $+1 \mathrm{~mm}$ anterior of bregma), and a $1.5 \times 1.5 \mathrm{~mm}$ region of skull overlying this region of cortex was thinned to $\sim 50 \%$ of original thickness using a high-speed dental drill. In the remaining mice $(n=18)$, imaging of IOSs through a thinnedskull imaging preparation was used to identify cortical regions activated by vibrotactile stimulation $(1 \mathrm{~s}, 100 \mathrm{~Hz})$ of the contralateral forelimb (cFL). (In both sets of animals, IOS imaging was performed before in vivo $\mathrm{Ca}^{2+}$ imaging.) Briefly, a $4 \times 4 \mathrm{~mm}$ region overlying the approximate location of S1FL and primary hindlimb somatosensory cortex (S1HL) was thinned slightly using a high-speed drill and smoothed using a rounded scalpel blade (Surgistar \#6400). This region was covered with $1.3 \%$ low-melt agarose (at $37-38^{\circ} \mathrm{C}$; Type 3-A; Sigma-Aldrich; A9793) dissolved in HEPES-buffered artificial CSF (ACSF), and sealed with a glass coverslip $(\# 1)$. Anesthetic concentration was reduced to $0.5-1.0 \%\left(50 \% \mathrm{O}_{2}, 50 \%\right.$ air $)$, and imaging of IOSs during limb stimulation was performed as described below. IOS response maps for $\mathrm{CFL}$ and contralateral hindlimb (cHL) were processed as described below, and the cFL representation was targeted for photothrombosis.

Photothrombosis. To induce targeted and highly reproducible cerebral infarctions of S1FL, we used a modified version of the photothrombotic method originally described by Watson et al. (1985). This model has been used previously in our laboratory in studies of acute and chronic structural plasticity after stroke (Zhang et al., 2005; Brown et al., 2007, 2008; Enright et al., 2007; Zhang and Murphy, 2007). As described above, S1FL was identified by stereotaxic coordinates or imaging of the cFL-evoked intrinsic signal in isoflurane-anesthetized animals ( $2 \%$ induction, $1.5 \%$ maintenance, in air). The skull overlying S1FL was thinned, and the surrounding skull was coated with black marker to prevent scattered light from entering into adjacent cortex during photothrombosis. Mice were given an injection of $1 \%(\mathrm{w} / \mathrm{v})$ solution of Rose Bengal (100 mg/kg, i.p.) in 0.01 м PBS. To photoactivate the Rose Bengal, S1FL was illuminated with a collimated beam of green laser light (532 $\mathrm{nm}, 17 \mathrm{~mW}$; diameter, $\sim 1.5 \mathrm{~mm}$ ) for $17 \mathrm{~min}$. Sham surgery controls were treated in an identical manner with the omission of either laser illumination or Rose Bengal injection. For images of the histological appearance of neurons after such a stroke, see Brown et al. $(2007,2008)$. After surgery, mice were returned to their home cages and were allowed to recover.

IOS image capture and processing. For imaging of IOSs, the cortical surface was illuminated by red or green light-emitting diodes (LEDs) mounted around the microscope objective and powered by a regulated DC power supply (Circuit Test). The green LED light source was used for visualizing the surface vasculature overlying the somatosensory cortex. The red LED light source $(635 \mathrm{~nm})$ was used for IOS imaging. Imaging acquisition was performed using XCAP-standard version 2.2 imaging software and frame grabber (EPIX) with a Dalsa 1M60 Pantera 12-bit 
camera mounted on a video macroscope setup that used a short focus front-to-front dual-video lens system $(3.8 \times 3.8 \mathrm{~mm}$ field, $7.5 \mu \mathrm{m}$ pixels $)$ (Grinvald et al., 1986; Bonhoeffer and Grinvald, 1996). The depth of focus was set to $200-400 \mu \mathrm{m}$ below the cortical surface.

Mechanical limb stimulation was achieved with custom-made mechanical limb manipulators incorporating piezoceramic bending actuators (Piezo Systems; \#Q220-AU-203YB). To image sensory-evoked IOSs, vibrotactile limb stimulation $(1 \mathrm{~s}, 100 \mathrm{~Hz})$ was alternated between $\mathrm{cHL}$ and cFL. For each limb, 40 trials were imaged at a frame rate of $10 \mathrm{~Hz}$ for $3.0 \mathrm{~s}$ (1.5 s before and after stimulus onset; interstimulus intervals = 20 s). In some cases, IOS imaging during stimulation of the ipsilateral limbs was also performed, although this was prohibited in most cases by the time constraints of the combined imaging protocol. The image acquisition, limb stimulation, and LED illumination were synchronized using transistor-transistor logic signals.

Image analysis was performed in Image J (NIH). Trials for each limb were first summed and mean filtered (radius 3). Frames $1.5 \mathrm{~s}$ before stimulation were summed to create a "blank" reference, and those during and after stimulation (over $1.5 \mathrm{~s}$ ) were summed to create a "response“ image. By dividing the response image by the blank reference image, an "IOS response map" could be made to identify the hindlimb- and forelimb-responsive cortex. These response maps could be thresholded at $50 \%$ of the maximal response and merged to create color-coded response maps delineating $\mathrm{CHL}$ - and cFL-responsive regions on the surface vasculature.

Poststroke imaging. After a specified recovery period (2 weeks, 1 month, or 2 months), mice were anesthetized with $15 \%(w / v)$ urethane dissolved in distilled water $(1.25 \mathrm{~g} / \mathrm{kg}$; supplemented at $0.25 \mathrm{~g} / \mathrm{kg}$ as needed). An acute imaging preparation using urethane anesthesia was used because bolus loading of calcium indicator necessitates an acute experiment. Urethane provides long-lasting, stable anesthesia for prolonged imaging experiments but does not permit recovery of the animal, necessitating the use of isoflurane during prestroke IOS imaging. A $4 \times 4$ $\mathrm{mm}$ piece of skull over the somatosensory cortex, including the S1HL, S1FL, and infarct, was thinned with a dental drill and removed with forceps, leaving the dura intact. The exposed brain was bathed in HEPESbuffered ACSF and covered with a glass coverslip (\#1). To minimize movement artifact, the skull was fastened with cyanoacrylate glue and dental cement to a stainless-steel chamber that was directly affixed into a metal plate that could be mounted onto the IOS macroscope and twophoton microscope.

IOS image acquisition was performed as described above and in previous papers from our laboratory (Brown et al., 2007; Winship et al., 2007; Zhang and Murphy, 2007), and response maps identifying regions activated by cHL or CFL stimulation were identified. After IOS imaging, the coverslip was removed, and small pressure injections (Picospritzer II; General Valve Corporation) of the membrane-permeant $\mathrm{Ca}^{2+}$ indicator Oregon Green BAPTA-1 (OGB-1; Invitrogen) were made $200 \mu \mathrm{m}$ below the cortical surface using glass micropipettes with resistances of $\sim 5 \mathrm{M} \Omega$ (Stosiek et al., 2003; Garaschuk et al., 2006). Wherever possible, microinjections of OGB-1 were made into both cFL- and cHL-responsive regions, as identified by the poststroke IOS response map. Subsequent to OGB-1 AM injection, the cortex was incubated with sulforhodamine 101 (SR101; Sigma-Aldrich) (Nimmerjahn et al., 2004) to facilitate identification of astrocytes. The craniotomy was then covered with $1.3 \%$ agarose dissolved in HEPES-buffered ACSF and sealed with a glass coverslip (\#1).

Two-photon imaging was performed using a modified Olympus BX50W1 upright microscope equipped with a Ti:Sapphire Coherent Mira 900 mode-locked laser tuned to $810 \mathrm{~nm}$ for OGB-1 and SR101 excitation [described previously by Zhang et al. (2005), Brown et al. (2007), and Zhang and Murphy (2007)]. Olympus IR-LUMPLanFl [40×; numerical aperture (NA), 0.8] and 60× Olympus LUMFL (NA, 1.1) water-immersion objectives were used. Images were acquired at $3.0-$ 11.6 Hz (mean, 6.6 Hz; SD, $1.3 \mathrm{~Hz}$ ) using custom software routines (IgorPro; Wavemetrics) interfacing with a 16-bit data acquisition card (PCI-6110; National Instruments). $\mathrm{Ca}^{2+}$ fluctuations in neurons, astrocytes, and regions of interest (ROIs) in the surrounding neuropil in layers 2/3 (100-200 $\mu \mathrm{m}$ below the cortical surface; mean depth, $137 \mu \mathrm{m}$; SD,
$23.9 \mu \mathrm{m}$ ) of the somatosensory cortex were imaged in response to 10 trials of brief $(1.0 \mathrm{~s}, 100 \mathrm{~Hz})$ mechanical stimulation of each limb.

Two-photon image processing. A median filter (radius, 1 pixel) was applied to each image to reduce background noise associated with photon or photomultiplier tube noise. Using custom IgorPro and ImageJ functions, $\mathrm{Ca}^{2+}$ transients in each trial of stimulation and mean traces of $\mathrm{Ca}^{2+}$ oscillation across 10 trials for each limb were made for individual neurons, astrocytes, and neuropil ROIs. Mean traces were imported into Clampfit 9.0 (Molecular Devices), and responding cells were identified using a threshold search. Decay and onset analysis and averaging of mean neuropil, neuronal, and image field transients were also performed in Clampfit 9.0. Thresholds were set to identify cells in which the change in $\mathrm{Ca}^{2+}$-induced fluorescence relative to baseline fluorescence was greater than one SD over baseline and remained above this criterion for at least $0.5 \mathrm{~s}$ (or $3 \times$ frame rate for frame rates $>170 \mathrm{~ms} /$ frame). Neurons or astrocytes with stimulus-evoked (onset coinciding with stimulus onset) transients that met these threshold criteria were considered responsive. These criteria were found effective for separating responsive cells from noise.

Statistical analyses. Multivariate comparisons were made using a multivariate ANOVA (MANOVA; Wilk's $\lambda$ test; SPSS 15.0). Univariate comparisons were made using ANOVA with post hoc Tukey's honestly significant difference (HSD) tests. A $p$ value $\leq 0.05$ was considering statistically significant. Data are expressed as the mean \pm SEM.

\section{Results}

\section{Imaging regional and single-cell somatosensory processing in vivo}

Figure 1 illustrates the imaging protocol and experimental timeline used to study regional and single-cell plasticity in the somatosensory cortex of adult mice after targeted ischemic insult. Briefly, vibrotactile stimulation $(1 \mathrm{~s}, 100 \mathrm{~Hz})$ of individual limbs (distal to the elbow/knee) during imaging of IOSs was used to measure the hemodynamic response to cortical activation and establish a map of responsive cortex. Limb stimulation in a naive mouse induces an "initial dip" in light reflectance in active regions (Fig. 1A), likely because of a relative increase in deoxyhemoglobin after stimulus onset (Grinvald et al., 1986; Frostig et al., 1990; Devor et al., 2003). The color-coded response map in Figure $1 A$ (right) illustrates regions of the somatosensory cortex activated by cHL (red shading) and cFL (green shading) stimulation, thresholded at $50 \%$ of peak amplitude.

IOS response maps were used to target regions of cortex to be injected with the single-cell $\mathrm{Ca}^{2+}$ indicator OGB-1 (Stosiek et al., 2003; Garaschuk et al., 2006). Both cHL and cFL representations were targeted for OGB-1 injection, and only data from mice in which both IOS and $\mathrm{Ca}^{2+}$ imaging data were acquired were included in our analyses. The astrocyte marker SR101 (Nimmerjahn et al., 2004) facilitated differentiation of neurons and astrocytes. Figure $1 B$ shows two-photon images of OGB-1 and SR10 1 labeling in layer 2 (depth, $147 \mu \mathrm{m}$ ) acquired from the area demarcated by the black box (B) in the region activated by $\mathrm{cHL}$ stimulation in Figure $1 A$. Neurons and astrocytes could be clearly differentiated and their sensory-evoked $\mathrm{Ca}^{2+}$ responses recorded in vivo via two-photon laser-scanning microscopy (Winship et al., 2007). In Figure $1 C$, the mean sensory-evoked increases in intracellular $\mathrm{Ca}^{2+}$ concentration $\left(\left[\mathrm{Ca}^{2+}\right]_{\mathrm{i}}\right)$, as indicated by the change in OGB-1 fluorescence divided by the baseline fluorescence $\left(\Delta F / F_{\mathrm{o}} \times 100\right)$, averaged from 10 trials of $\mathrm{cHL}$ stimulation $(1 \mathrm{~s}, 100 \mathrm{~Hz})$, are illustrated for two neuronal somata, one astrocyte soma, and one ROI in the neuropil (areas devoid of cell bodies) (Fig. $1 B$ ). Previous studies have demonstrated that $\mathrm{Ca}^{2+}$ transients in the neuronal somata reflect $\left[\mathrm{Ca}^{2+}\right]_{i}$ elevation caused by action potential firing, rather than subthreshold activity (Mao et al., 2001; Stosiek et al., 2003; Berger et al., 2007; Sato 


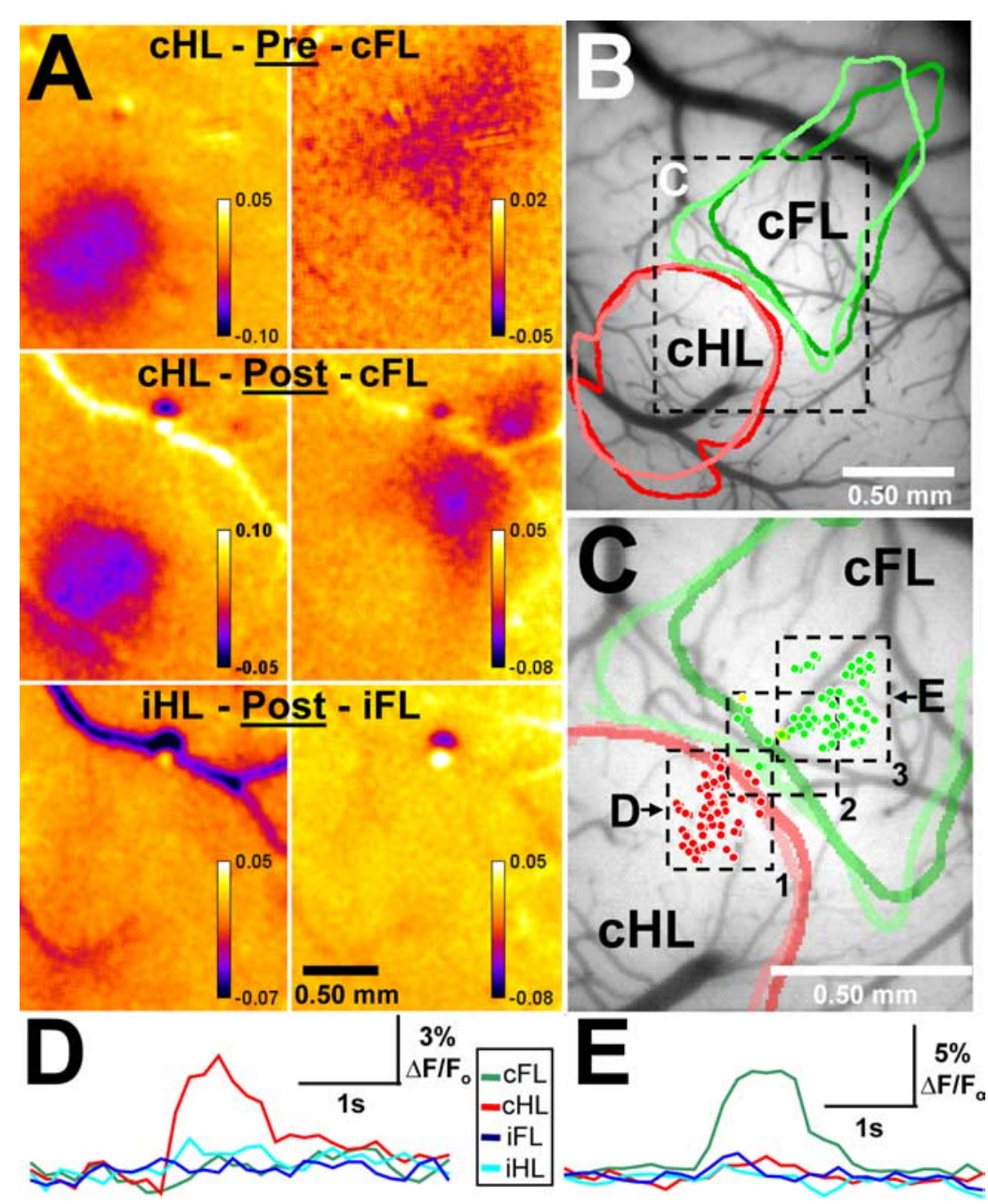

Figure 2. Regional and cellular organization of forelimb and hindlimb somatosensory cortex. $\boldsymbol{A}$, IOS response maps in a control mouse before and after the sham procedure reveal a consistent topography of CHL-and CFL-activated cortex, with CHL-activated tissue posterior and medial to the CFL representation. Mechanical stimulation of the ipsilateral limbs did not elicit a clear region of activated cortex. $\boldsymbol{B}$, The borders of the presham and postsham IOS response maps from $\mathrm{CHL}$ and $\mathrm{CFL}$ stimulation, thresholded at $50 \%$ of peak response amplitude (light red/green lines demonstrated presham borders; dark $\mathrm{red} / \mathrm{green}$ lines demonstrated postsham borders). Despite different imaging conditions and anesthesia, IOS response maps collected before and after sham procedures revealed consistent patterns. C, Two-photon $\mathrm{Ca}^{2+}$ imaging was performed at three locations (1-3, demarcated by dashed boxes) spanning the border between CHL- and CFL-activated cortex (three depths per location: 120,138, and $156 \mu \mathrm{m}$ below the cortical surface). A threshold analysis of somatic responses was performed for these image planes, and the locations of neurons with above-threshold responses to limb stimulation are superimposed on the map of the IOS response borders. Green dots show the locations of neurons with above-threshold responses to $\mathrm{CFL}$ stimulation, and red dots show the locations of $\mathrm{CHL}$-activated neurons. Only two neurons responded to stimulation of any of the other limbs: one neuron responded to iFL stimulation alone (yellow dot), and a second neuron responded to both forelimbs (green dot, yellow border). Importantly, two-photon imaging revealed an extremely sharp border at the cellular level, with no spatial overlap between neurons sensitive to $\mathrm{CHL}$ or CFL stimulation. $\boldsymbol{D}, \boldsymbol{E}$, Representative image field traces from image planes ( $\boldsymbol{D}$, depth, $120 \mu \mathrm{m}$; $\boldsymbol{E}$, depth, $138 \mu \mathrm{m}$ ) adjacent to the border in $\mathrm{CHL}$ - and CFL-activated cortex, respectively (depth, 120 and 128).

et al., 2007), whereas transients in the neuropil are predominantly nonsomatic and in part reflect presynaptic activity (Kerr et al., 2005). In neurons $\mathrm{n} 1$ and $\mathrm{n} 2$ and neuropil ROI np1, $\mathrm{Ca}^{2+}$ fluorescence increased within one sample after cHL stimulation onset, although differences in amplitude and duration are apparent. Astrocyte al had no stimulus-evoked activity. Single-trial responses are illustrated for $\mathrm{n} 1$ and $\mathrm{n} 2$ in supplemental Figure 1 (available at www.jneurosci.org as supplemental material).

This imaging protocol, progressing from macroscopic imaging of sensory-evoked IOSs to single-cell resolution with two- photon $\mathrm{Ca}^{2+}$ imaging, was used to characterize sensory processing in control animals and animals after targeted photothrombotic infarction of the S1FL according to the experimental timeline shown in Figure $1 \mathrm{D}$. In isofluraneanesthetized animals, S1FL was targeted for photothrombosis (or sham procedure) via imaging of sensory-evoked IOSs ( $n=18$ mice) or stereotaxic coordinates $[n=12$ mice; coordinates from Paxinos and Franklin (2001) and confirmed by in vivo $\mathrm{Ca}^{2+}$ imaging]. Consistent with previous studies from our laboratory (Brown et al., 2007), this stroke protocol allowed for highly reproducible targeted lesions of S1FL cortex (lesion diameter, 0.5-1.0 $\mathrm{mm}$ ). After photothrombosis (or sham), animals recovered in their home cages for 2 weeks, 1 month, or 2 months. After the recovery period, imaging of sensory-evoked IOSs and $\mathrm{Ca}^{2+}$ signals were performed through a cranial window in urethane-anesthetized adult mice (Brown et al., 2007; Winship et al., 2007).

\section{Limb selectivity in the somatosensory cortex of control mice}

In naive and sham control mice $(n=12)$, imaging of IOSs revealed a highly consistent spatial relationship between regions of the somatosensory cortex activated by $\mathrm{cHL}$ and $\mathrm{cFL}$ stimulation. As illustrated in Figures $1 A$ and 2, $A$ and $B$, brief vibrotactile stimulation of the distal cHL activated a region of cortex immediately posterior and medial to regions activated by cFL stimulation. Importantly, the transition from cFL- to cHL-activated regions consisted of a sharp border with little overlap between the IOS representations [visible in both the raw (Fig. 2A) and thresholded (Fig. 2B) maps]. The $\mathrm{cHL}$ and $\mathrm{cFL}$ representations defined by the IOSs closely match the S1FL and S1HL somatosensory regions in the atlas of Paxinos and Franklin (2001), and no differences were observed between naive animals and sham controls. Stimulation of the ipsilateral limbs produced little or no activation in the IOS response maps (Fig. 2A). Despite differences in imaging conditions (thin-skull vs cranial window) and anesthetic (isoflurane vs urethane), cFL- and cHL-responsive regions defined by the IOS response maps acquired before sham procedures and after recovery (in sham groups) were highly consistent (Fig. 2A,B).

Color coding of neuronal somata by limb preference permitted the creation of topographical maps showing the spatial organization of responsive neurons. Two-photon $\mathrm{Ca}^{2+}$ imaging spanning the border of the S1HL and S1FL representations in control mice $(n=3)$ was performed to determine the fine cellular topography underlying the borders between functional domains that were suggested by the IOSs. The mean $\left[\mathrm{Ca}^{2+}\right]_{\mathrm{i}}$ elevations in individual neurons near the S1FL/S1HL border during 10 trials of stimulation of each limb were determined, and threshold criteria (see Materials and Methods) were used to separate responsive cells from background noise and de- 


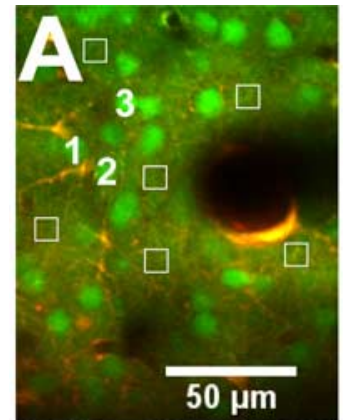

S1HL - Depth 147

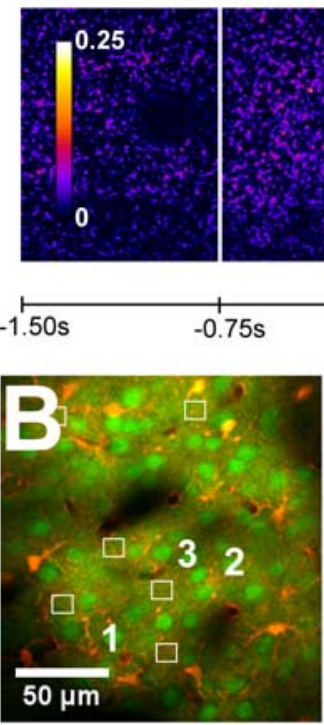

S1FL - Depth 114

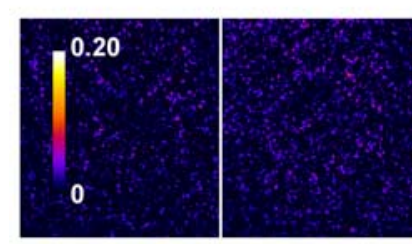

$-1.56 \mathrm{~s}$

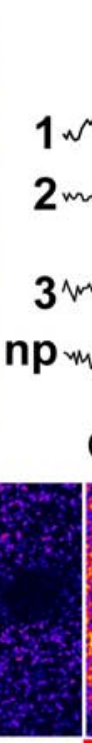

1 mammenming

$\bigcap_{M}$<smiles>C1CCCC1</smiles>

.

2 man

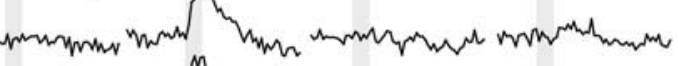

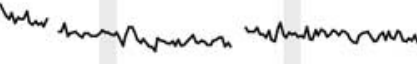

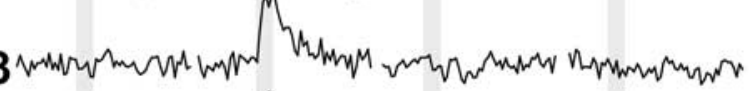

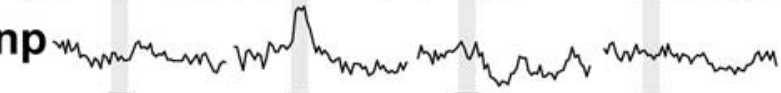
$c \bar{F} \mathrm{c} \overline{\mathrm{H} L} \quad \mathrm{i} \overline{\mathrm{F} L} \quad \mathrm{i} \overline{\mathrm{H}} \mathrm{F}$
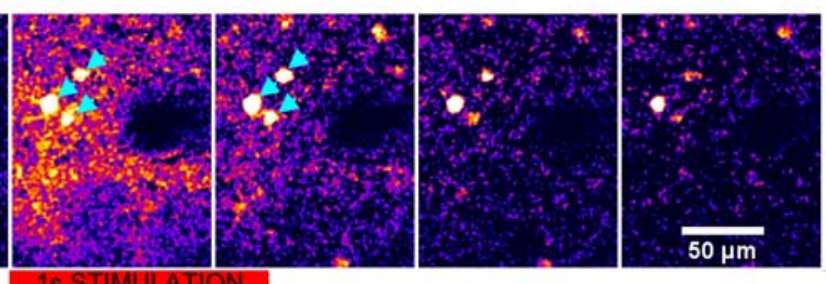

$0 \mu \mathrm{m}$

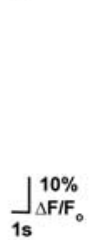

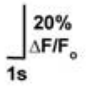

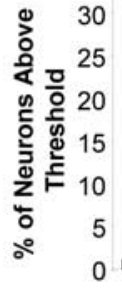

Mean Image Field Activity in S1HL
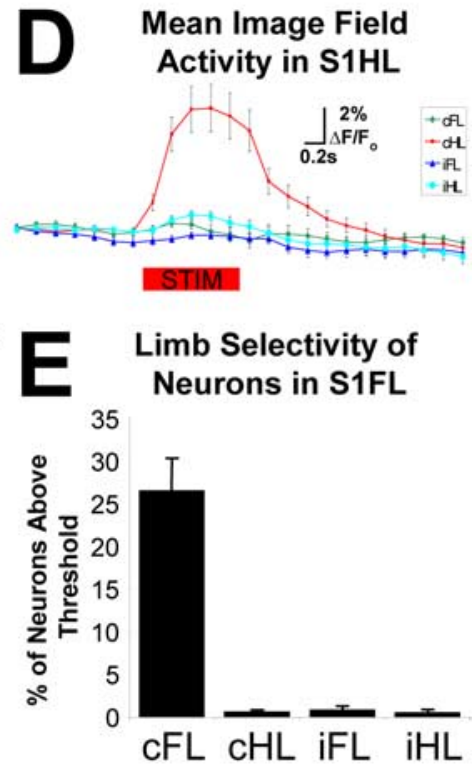

\rfloor$_{\Delta \mathrm{s}}^{10 \% \mathrm{~F}}$ 。

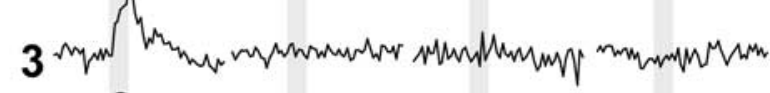

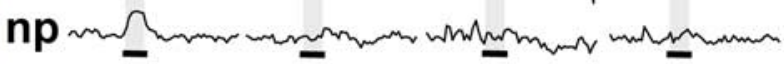

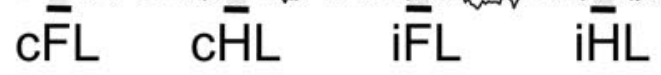
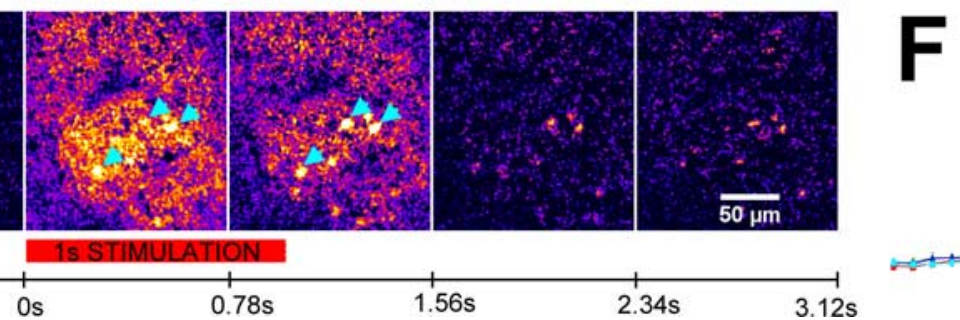

Mean Image Field Activity in S1FL

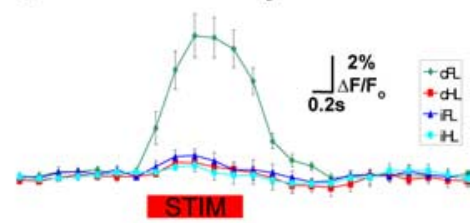

Figure 3. Limb selectivity in somatosensory cortex of control mice. $A$, Two-photon data from layer $2 / 3$ of the $S 1 \mathrm{HL}$ of a naive mouse, from the region defined by the black box in Figure $1 A$. Mean $\mathrm{Ca}^{2+}$ signals (average of 10 trials of stimulation per limb) from three neurons (1-3, labeled in the 0GB-1/SR101 image on the left) and the mean neuropil (np) response from six areas of the np (white boxes) are shown. Sensory-evoked $\mathrm{Ca}^{2+}$ transients were only elicited by stimulation of the $\mathrm{CHL}$ [gray shading denotes period of vibrotactile stimulation ( $\left.1 \mathrm{~s}, 100 \mathrm{~Hz}\right)$ ]. Difference image montages (bottom) show the responding neuronal somatic $\mathrm{Ca}^{2+}$ signal, and were created by binning in groups of five frames (by averaging) and subtracting the mean baseline image from each frame. The difference images in $A$ illustrate the change in fluorescence $\left(\Delta F / F_{0}\right)$ from baseline fluorescence over $0.75 \mathrm{~s}$ before, during, and after $\mathrm{CHL}$ stimulation. $\boldsymbol{B}$, Two-photon traces and difference images from S1FL (depth, $114 \mu \mathrm{m}$ ) of a control mouse. Again, only stimulation of the preferred limb (cFL) elicited a strong $\mathrm{Ca}^{2+}$ transient. Threshold criteria (see Materials and Methods) were used to define "strong" somatic responses in neurons in S1HL and S1FL. Graphs in C and $\boldsymbol{E}$ show the prevalence of above-threshold responses to limb stimulation in S1HL (2282 neurons) and S1FL (1498 neurons), respectively, in control animals. Selectivity for the preferred limb and a paucity of responses to mechanical stimulation of the other limbs is apparent. $\boldsymbol{D}, \boldsymbol{F}$, The mean image field $\mathrm{Ca}^{2+}$ transient records across all control animals (S1HL, 9 mice; S1FL, 10 mice), analogous to a local sensory-evoked field recording. Representative image field signals in response to stimulation of each limb were acquired from each animal and averaged (error bars show the SEM). Again, nearly complete selectivity for the preferred limb is apparent.

termine the prevalence of sensory-evoked $\mathrm{Ca}^{2+}$ transients in the layer $2 / 3$ somata according to limb. Figure $2 C$ shows representative data from a sham control (Rose Bengal injection without photoactivation, 2 week recovery). Above-threshold responses to limb stimulation in layer $2 / 3$ neurons spanning the transition zone between S1HL and S1FL IOS maps (Fig. $2 B$ ) are superimposed onto a map of the surface vasculature. Red and green dots show the locations of neurons with above-threshold responses to $\mathrm{cHL}$ and $\mathrm{cFL}$ stimulation, respectively. Imaging was performed on image sections at three depths $(120,138$, and $156 \mu \mathrm{m}$ from the cortical surface) in the S1FL (labeled as location 3), spanning the S1FL/S1HL border (location 2), and in the S1HL (location 1; borders of the image sections are outlined by the black dashed lines in Fig. 2C). With the exception of two neurons in S1FL, which responded to ipsilateral forelimb (iFL) stimulation alone (yellow dot) or both cFL and iFL stimulation (green with yellow border), neuronal somata in S1FL and S1HL were completely selective for their preferred limb as defined by IOS mapping. Furthermore, two-photon imaging confirmed a precise cellular architecture in primary somatosensory cortex, with a sharp bor- 
Table 1. Amplitude and time to peak of above-threshold somatic responses in S1FL

\begin{tabular}{|c|c|c|c|c|c|c|c|c|}
\hline \multirow[b]{2}{*}{ Group } & \multicolumn{4}{|l|}{ Amplitude $\left(\Delta F / F_{0}, \%\right)$} & \multicolumn{4}{|c|}{ Time to peak from stimulus onset (s) } \\
\hline & Preferred limb (CFL) & SEM & Nonpreferred limbs & SEM & Preferred limb (cFL) & SEM & Nonpreferred limbs & SEM \\
\hline Control & 14.1 & 2.4 & 10.8 & 1.3 & 0.63 & 0.04 & 0.69 & 0.11 \\
\hline 1 Mth & 9.9 & 0.7 & 9.4 & 1.3 & 0.61 & 0.05 & 0.57 & 0.06 \\
\hline 2 Mths & 14.7 & 3.6 & 10.4 & 2.5 & 0.63 & 0.06 & 1.03 & 0.13 \\
\hline
\end{tabular}

Mth, Month.

Table 2. Amplitude and time to peak of above-threshold somatic responses in S1HL

\begin{tabular}{|c|c|c|c|c|c|c|c|c|}
\hline \multirow[b]{2}{*}{ Group } & \multicolumn{4}{|l|}{ Amplitude $\left(\Delta F / F_{0}, \%\right)$} & \multicolumn{4}{|c|}{ Time to peak from stimulus onset (s) } \\
\hline & Preferred limb (cHL) & SEM & Nonpreferred limbs & SEM & Preferred limb (cHL) & SEM & Nonpreferred limbs & SEM \\
\hline Control & 13.5 & 2.1 & 11.5 & 1.9 & 0.61 & 0.02 & 0.77 & 0.11 \\
\hline $2 \mathrm{Wks}$ & 12.0 & 1.6 & 9.2 & 3.4 & 0.59 & 0.01 & 0.42 & 0.11 \\
\hline $1 \mathrm{Mth}$ & 12.7 & 2.4 & 14.0 & 3.0 & 0.51 & 0.02 & 0.73 & 0.05 \\
\hline 2 Mths & 12.6 & 1.9 & 13.8 & 3.6 & 0.62 & 0.07 & 0.61 & 0.10 \\
\hline
\end{tabular}

Wks, Weeks; Mth, month.

der between S1FL and S1HL and no overlap between CHL- and cFL-responsive neuronal somata.

Figure 3 shows representative two-photon images of OGB-1 and SR101 labeling, traces of sensory-evoked $\mathrm{Ca}^{2+}$ transients, and "difference image" montages illustrating the $\mathrm{Ca}^{2+}$ response to limb stimulation in S1HL (Fig. 3A) (showing the same image section illustrated in Fig. 1) and S1FL (Fig. 3B) (IOS data not shown) in a naive mouse and a sham control (Rose Bengal without laser illumination, 2 month recovery), respectively. Traces show the mean $\left[\mathrm{Ca}^{2+}\right]_{\mathrm{i}}$ elevation from 10 trials of stimulation of each limb for the representative neurons demarcated in the OGB1/SR101 images and the mean neuropil response from the regions defined by the white boxes. Strikingly, neurons in the primary S1HL and S1FL cortex were highly limb selective, and sensory-evoked $\mathrm{Ca}^{2+}$ transients were elicited only by stimulation of the $\mathrm{cHL}$ and $\mathrm{cFL}$, respectively (Fig. $3 A, B$, traces). Difference image montages in Figure 3 illustrate the change in fluorescence $\left(\Delta F / F_{\mathrm{o}}\right)$ from baseline fluorescence over $0.75 \mathrm{~s}$ (Fig. $3 A$ ) or $0.78 \mathrm{~s}$ (Fig. $3 B$ ) before, during, and after cHL (Fig. $3 A$ ) or cFL (Fig. $3 B$ ) stimulation. Difference images illustrate the mosaic pattern of responding neuronal somata (Stosiek et al., 2003; Ohki et al., 2005), and the varied amplitude and duration of these responses. Note that an increase in neuropil fluorescence is apparent in the difference images after stimulation as a relatively homogenous change in background.

The response properties of 3780 neurons in the S1HL (2282 neurons; 9 mice total: 3 naive, 4 laser without Rose Bengal sham controls, and 2 Rose Bengal without laser shams) and S1FL (1498 neurons; 10 mice total: 3 naive, 4 laser without Rose Bengal shams, and 3 Rose Bengal without laser shams) of control mice were recorded in vivo. Threshold criteria determine the prevalence of sensory-evoked $\mathrm{Ca}^{2+}$ transients neuronal somata. The prevalence of cFL-, cHL-, iFL-, and ipsilateral hindlimb (iHL)evoked $\mathrm{Ca}^{2+}$ responses in control mice are illustrated for S1HL and S1FL in Figure 3, $C$ and $E$, respectively. Twenty-five to thirty percent of neurons exhibited an above-threshold, stimulusevoked response to the preferred limb ( $\mathrm{cHL}$ in S1HL $=27.3 \pm$ $2.0 \%$; cFL in $\mathrm{S} 1 \mathrm{FL}=26.5 \pm 3.8 \%$ ). The mean peak amplitudes $\left(\Delta F / F_{\mathrm{o}}\right)$ and time to peak from stimulus onset for abovethreshold $\left[\mathrm{Ca}^{2+}\right]_{\mathrm{i}}$ signals in S1FL and S1HL are listed in Tables 1 and 2, respectively. Preferred-limb responses in control mice reached peak amplitude $\left(\sim 14 \% \Delta F / F_{\mathrm{o}}\right) \sim 0.6 \mathrm{~s}$ after stimulus onset. Sensory-evoked $\mathrm{Ca}^{2+}$ signals in neurons in the barrel cortex of mice and rats exhibit peak amplitudes 15-30\% above base- line with response probabilities $<0.5$ for a given trial (Kerr et al., 2007; Sato et al., 2007; Dombeck et al., 2007), and studies in the primary visual cortex of rats suggest that $25-75 \%$ of neurons respond to visual stimulation with signal amplitudes up to $30 \%$ above baseline (Ohki et al., 2005). Given that our 10 trial averages would, in many cases, include both responsive and nonresponsive trials (supplemental Fig. 1, available at www.jneurosci.org as supplemental material), the incidence and amplitude of sensoryevoked $\mathrm{Ca}^{2+}$ responses reported here agree well with these previous studies. Additionally, Kerr et al. (2005) performed cellattached recordings during two-photon $\mathrm{Ca}^{2+}$ imaging with OGB-1 and reported that single action potentials generated a $\mathrm{Ca}^{2+}$ transient with a peak amplitude of $\sim 10 \%$, whereas two or more action potentials generated transients with amplitudes of $>15 \%$. As such, our threshold criteria appear appropriate for detecting neurons responding to limb stimulation by firing at least one action potential. Consistent with our previous work (Winship et al., 2007), the neuropil (containing putatively presynaptic signals) (Kerr et al., 2005) exhibited more rapid decay kinetics than neuronal somata (Table 3). Above-threshold responses to stimulation of the nonpreferred limbs (within a map territory defined by IOS imaging) were rare (S1HL: $\mathrm{cFL}=1.3 \pm$ $0.4 \%, \mathrm{iFL}=0.3 \pm 0.1 \%, \mathrm{iHL}=1.2 \pm 0.5 \%$; S1FL: $\mathrm{cHL}=0.7 \pm$ $0.2 \%, \mathrm{iFL}=0.9 \pm 0.3 \%, \mathrm{iHL}=0.6 \pm 0.3 \%)$. Neither peak amplitude nor time to peak for aberrant responses differed significantly from preferred-limb responses, although there was a trend toward reduced peak amplitude for aberrant limb responses in both S1HL and S1FL (Tables 1,2).

Selectivity for the preferred limb (based on IOS maps) is also apparent when examining sensory-evoked $\left[\mathrm{Ca}^{2+}\right]_{\mathrm{i}}$ elevations in the neuropil alone and the mean "image field" signal in control animals. As noted, neuropil activity provides a measure of predominantly nonsomatic (and putatively presynaptic) activity (Kerr et al., 2005). Threshold analysis of the mean sensoryevoked neuropil transients in S1HL (Table 4) and S1FL (Table 5) demonstrated that preferred-limb stimulation elicited abovethreshold transients at all imaging depths assessed in control animals, whereas above-threshold responses to the nonpreferred limbs were rare. Image field transients illustrate the mean $\left[\mathrm{Ca}^{2+}\right]_{i}$ increase across the entire two-photon image field, pooling axonal, dendritic, and somatic signals, and provide a measure of local network activity analogous to an evoked field potential. Mean image field activity from all control animals is shown in Figure 3, $D$ (S1HL) and $F(\mathrm{~S} 1 \mathrm{FL})$ : again, selectivity for the pre- 
Table 3. Decay $\tau$ of somatic and neuropil transients

\begin{tabular}{|c|c|c|c|c|c|c|c|c|}
\hline \multirow[b]{2}{*}{ Group } & \multicolumn{4}{|l|}{ S1FL } & \multicolumn{4}{|l|}{ S1HL } \\
\hline & Somatic & SEM & Neuropil & SEM & Somatic & SEM & Neuropil & SEM \\
\hline Control & 0.94 & 0.13 & 0.64 & 0.09 & 1.16 & 0.38 & 0.84 & 0.07 \\
\hline 2 Wks & - & - & - & - & 1.20 & 0.41 & 0.83 & 0.22 \\
\hline 1 Mth & 1.09 & 0.26 & 0.90 & 0.08 & 1.22 & 0.35 & 0.89 & 0.16 \\
\hline 2 Mths & 0.99 & 0.18 & 0.74 & 0.14 & 1.28 & 0.20 & 0.89 & 0.09 \\
\hline
\end{tabular}

Wks, Weeks; Mth, month.

Table 4. Above-threshold neuropil transients in S1HL

\begin{tabular}{|c|c|c|c|c|c|c|}
\hline \multirow[b]{2}{*}{ Group } & \multirow[b]{2}{*}{$n$ (mice) } & \multirow[b]{2}{*}{ Image planes } & \multicolumn{4}{|c|}{ Limbs stimulated during $\mathrm{Ca}^{2+}$ imaging in $\mathrm{S} 1 \mathrm{HL}$} \\
\hline & & & $\mathrm{CHL}$ & $\mathrm{CFL}$ & iFL & $\mathrm{iHL}$ \\
\hline Control & 9 & 23 & $23(100 \%)$ & $1(3.7 \pm 3.7 \%)$ & 0 & 0 \\
\hline $2 \mathrm{Wks}$ & 4 & 15 & $15(100 \%)$ & $1(5.0 \pm 5.0 \%)$ & 0 & $6(37.8 \pm 7.7 \%)$ \\
\hline 1 Mth & 6 & 15 & $15(100 \%)$ & $8(48.7 \pm 16.7 \%)$ & $1(8.3 \pm 8.3 \%)$ & $11(68.0 \pm 17.3 \%)$ \\
\hline 2 Mths & 6 & 19 & $19(100 \%)$ & $5(25.0 \pm 12.0 \%)$ & 0 & $4(23.7 \pm 11.9 \%)$ \\
\hline
\end{tabular}

For each limb, the number of image planes that exhibited a mean neuropil transient that met threshold criteria in S1HL is listed. The number in parentheses is the percentage of image sections (per animal \pm SEM) with above-threshold neuropil transient during stimulation of the specified limb. Wks, Weeks; Mth, month.

Table 5. Above-threshold neuropil transients in S1FL

\begin{tabular}{|c|c|c|c|c|c|c|}
\hline \multirow[b]{2}{*}{ Group } & \multirow[b]{2}{*}{$n$ (mice) } & \multirow[b]{2}{*}{ Image planes } & \multicolumn{4}{|c|}{ Limbs stimulated during $\mathrm{Ca}^{2+}$ imaging in S1FL } \\
\hline & & & $\mathrm{CHL}$ & $\mathrm{CFL}$ & iFL & iHL \\
\hline Control & 10 & 20 & $2(13.3 \pm 10.2 \%)$ & $20(100 \%)$ & 0 & 0 \\
\hline 1 Mth & 6 & 15 & $7(52.8 \pm 17.3 \%)$ & $15(100 \%)$ & 0 & $2(13.8 \pm 9.0 \%)$ \\
\hline 2 Mths & 4 & 10 & $3(33.3 \pm 11.8 \%)$ & $10(100 \%)$ & $2(25.0 \pm 25.0 \%)$ & $2(20.8 \pm 12.5 \%)$ \\
\hline
\end{tabular}

For each limb, the number of image planes that exhibited a mean neuropil transient that met threshold criteria in S1FL is listed (top number). The bottom number (in brackets) is the percentage of image sections (per animal \pm SEM) with above-threshold neuropil transient during stimulation of the specified limb. Mth, Month.

ferred limb and a paucity of responses to aberrant limbs is apparent. Image field activity on either side of the S1FL/S1HL border also supported the presence of a sharp functional border (Fig. $2 D, E)$.

\section{Disruption of S1FL activity and preserved S1HL activity 2 weeks after targeted stroke}

IOS response maps acquired from the same mouse before and after targeted infarction of S1FL are shown in Figure $4 A$. Before stroke, S1HL and S1FL exhibit a stereotypical configuration with a sharp border between the representations. After targeted thrombosis, the S1FL representation is disrupted, whereas S1HL activation remains. The borders of the prestroke and poststroke IOS maps (thresholded at $50 \%$ of the maximal response) are delineated on an image of the surface vasculature in Figure $4 \mathrm{~A}$ (bottom right). S1HL is truncated laterally by the infarction, whereas no clear S1FL activation is present. Preservation of the cHL-evoked representation suggests that functional hemodynamics are not disrupted in the peri-infarct cortex and the lack of cFL-evoked activity is not attributable to hemodynamic dysfunction. Additionally, recent work imaging sensory-evoked activity after stroke using voltage-sensitive dyes (a direct measure of depolarization) and IOSs in the same animals revealed remarkably similar patterns in the voltage-sensitive dye response and IOS maps (C. E. Brown and T. H. Murphy, unpublished observations). Disruption of cFL-evoked IOSs was characteristic of all mice imaged at 2 weeks after stroke $(n=4)$ (see also supplemental Fig. 2, available at www.jneurosci.org as supplemental material). $\mathrm{Ca}^{2+}$ imaging data from the region demarcated by the white box in Figure $4 A$ (bottom right) are shown in Figure $4 B$. The representative $\Delta F / F_{\mathrm{o}}$ traces and neuronal response map (depth, $126 \mu \mathrm{m})$ show that sensory-evoked activity in neuronal cell bodies and the surrounding neuropil was elicited by stimulation of the $\mathrm{cHL}$, with little or no activity in response to stimulation of the other limbs. An animal was classified as having altered limb selectivity if the summed prevalence of responses to the three nonpreferred limbs was two SDs greater than the prevalence of the nonpreferred limbs in the corresponding region of control animals. In three of four animals at 2 weeks after stroke, single-cell response properties within S1HL (to nonpreferred limbs) were comparable with controls and were not altered by targeted S1FL stroke. $\mathrm{Ca}^{2+}$ imaging revealed altered limb selectivity (above our criterion) in S1HL in one mouse, and representative data from this mouse are shown in supplemental Figure 2 (available at www.jneurosci.org as supplemental material). IOS data revealed that the S1FL representation was disrupted, whereas $\mathrm{Ca}^{2+}$ imaging (depth, $126 \mu \mathrm{m}$ ) identified a moderate increase in neuropil and somatic (supplemental Fig. $2 B, C$, available at www. jneurosci.org as supplemental material) responses to the nonpreferred limbs (demarcated by red arrowheads). Overall, threshold analysis of data from the S1HL of mice 2 weeks after FL-targeted stroke suggested a trend toward an increase in the number of neurons responding to cHL stimulation $(42.4 \pm 9.5 \%$ of 1300 neurons), with the prevalence of responses to aberrant limbs comparable with controls $(\mathrm{cFL}=1.3 \pm 0.5 \%$; $\mathrm{iFL}=0.4 \pm 0.2 \%$; $\mathrm{iHL}=2.5 \pm 1.5 \%)$. Nonsomatic activity in the neuropil (Table 4 ) in layer $2 / 3$ of the S1HL appears moderately altered 2 weeks after stroke, as evidenced by the increased incidence of abovethreshold transients in the neuropil after iHL stimulation.

\section{Functional plasticity after 1-2 months of recovery from targeted stroke}

Two weeks after targeted stroke of S1FL, no regional cFL-evoked activation was apparent in the IOSs, whereas macroscopic and microscopic imaging demonstrated that sensory processing was preserved in S1HL. In mice recovered for 1 month $(n=7)$ or 2 months $(n=7)$, however, regional and single-cell imaging revealed functional plasticity in both S1HL and S1FL. Representa- 


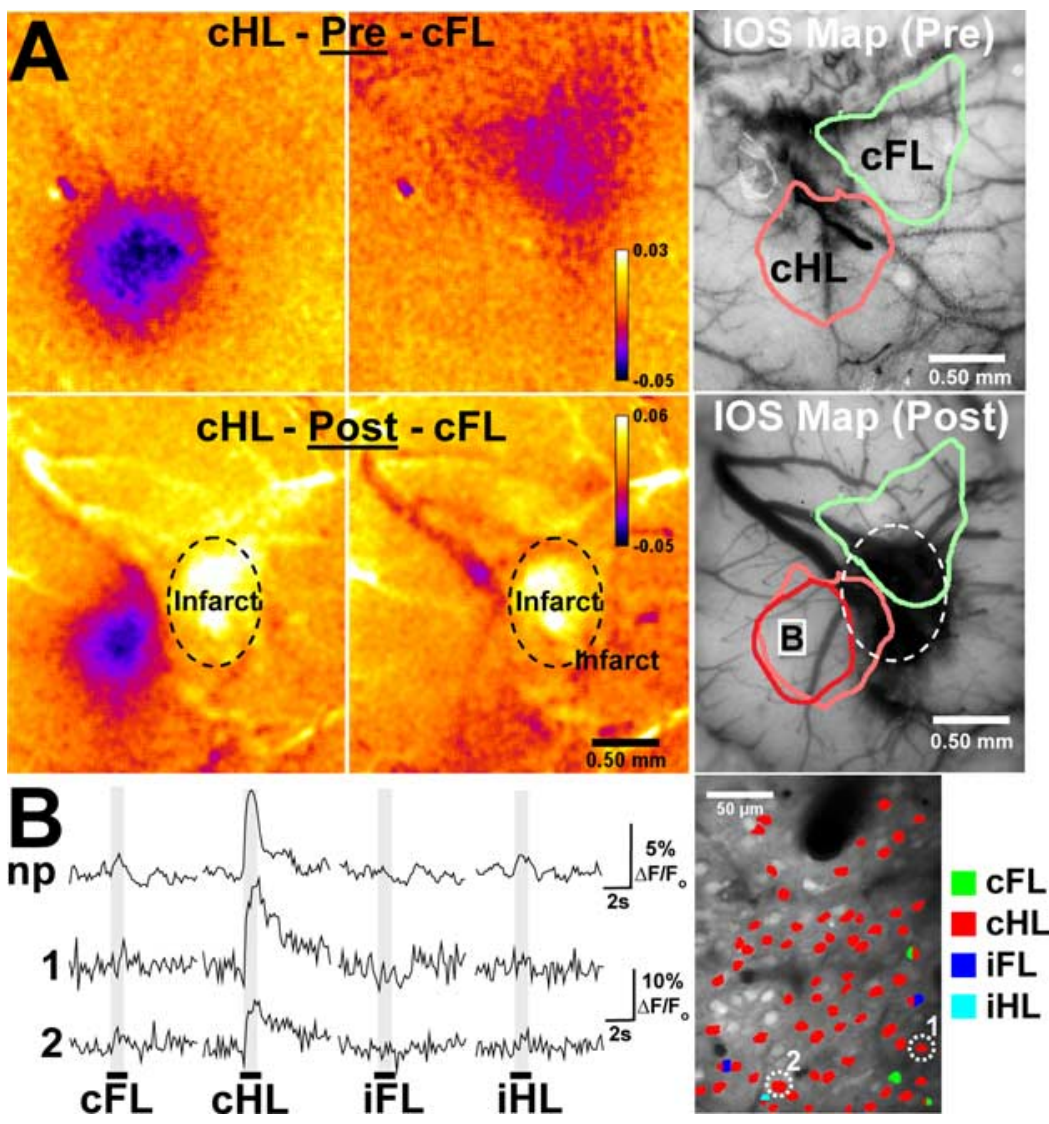

Figure 4. Disrupted forelimb representation and preserved hindlimb somatosensory processing 2 weeks after targeted stroke. $\boldsymbol{A}$, Prestroke and poststroke IOS response maps reveal disruption of the CFL representation 2 weeks after photothrombosis of S1FL. The thresholded IOS response map at the top right of $\boldsymbol{A}$ shows the limb representation before stroke, with the prestroke and poststroke maps merged onto a single image immediately below. No clear cFL-evoked activity was observed after stroke (in the original $\mathrm{FL}$ area), although the $\mathrm{CHL}$ representation was preserved (truncated laterally by the infarct). Weak CFL activation posterior to S1HL may represent a shifted pattern of cFLevoked activity, but its proximity to the edge of the craniotomy makes this difficult to discern. $\boldsymbol{B}$, Two-photon $\mathrm{Ca}^{2+}$ imaging in layer $2 / 3$ of the preserved $\mathrm{CHL}$ representation revealed normal limb selectivity in neurons and the surrounding neuropil in this animal. Representative data from individual neurons $(1,2)$ and the mean neuropil $(n p) \mathrm{Ca}^{2+}$ signal (average of 10 trials per limb, depth of $126 \mu \mathrm{m}$, from the region demarcated in $\boldsymbol{A}$ ) demonstrate normal sensoryevoked transients and preserved limb selectivity in S1HL. The color-coded neuronal response map (determined by threshold analysis of somatic responses, with color representing above-threshold responses to stimulation of the corresponding limb) demonstrates highly limb-selective responses to $\mathrm{CHL}$ stimulation in this image plane.

of IOS overlap demarcated by the black box in Figure $5 B$. In Figure $5 C$, representative traces from the image plane depicted in Figure $5 E$ (depth, $145 \mu \mathrm{m}$ ) show altered limb selectivity (red arrowheads) in the region of overlap. In the neuropil, clear, abovethreshold $\mathrm{Ca}^{2+}$ transients were elicited by stimulation of all four limbs. Similarly, neurons exhibiting above-threshold responses to stimulation of all four limbs (neuron 1) or both contralateral limbs (neuron 2) were also frequently observed; nonselective responses such as these were exceedingly rare in control animals. Single neuron somatic response maps from this region of S1HL/S1FL overlap are shown in Figure 5,D and E. Reduced limb selectivity is apparent, because above-threshold responses to stimulation of all limbs are represented within the maps, and many neurons respond to more than one limb. This altered neuronal organization (defined by somatic $\mathrm{Ca}^{2+}$ transients) contrasts with the clearly defined border of control animals (Fig. 2C), as well as S1HL maps at 2 weeks after stroke (Fig. $4 B$ ).

Figures $5, F$ and $G$, and $6 A-D$ show representative data from mice imaged 1 (Str25) and 2 months (Str34) after stroke, respectively. In both animals, cFL-evoked IOS activation appears shifted posterior and medial relative to control animals, with significant overlap with the S1HL region (yellow shading). In Figure 5G, neuronal response maps (depth, $135 \mu \mathrm{m}$ in S1HL core, $132 \mu \mathrm{m}$ in overlap region) show that limb selectivity was altered throughout S1HL, but most pronounced in regions of cFL/cHL IOS map overlap. In particular, note the abundance of individual neurons exhibiting above-threshold $\mathrm{Ca}^{2+}$ responses to stimulation of more than one limb in the overlapping region. $\mathrm{Ca}^{2+}$ imaging confirmed that neurons in layer $2 / 3$ (depth, $135 \mu \mathrm{m}$ ) of the most posterior regions of the reorganized cFL-activated cortex exhibited cFL-evoked activity. Within the posterior cFL-evoked activation in mouse Str34 (Fig. 6A$D), \mathrm{Ca}^{2+}$ imaging (depth, $141 \mu \mathrm{m}$ ) again confirmed that single neurons were strongly respon-

tive data from two animals are shown on the left side of Figure 5. Figure 5, $A$ and $B$, shows IOS data from mouse Str22, imaged 1 month after photothrombotic infarction of S1FL. Before photothrombosis, S1FL and S1HL exhibited a typical organization, separated by a relatively sharp border defined by IOS imaging. After stroke, CHL stimulation evokes activity in the same region of cortex (by IOSs), whereas cFL stimulation elicits a new regional pattern of activity. Figure $5 B$ shows the merge of the thresholded prestroke and poststroke IOS maps (prestroke maps outlined in light red and green, poststroke outlined with darker red and green borders and transparent shading). In this merged IOS image, it is apparent the infarction occupies the center of the prestroke S1FL and that the infarct core is not activated by cFL stimulation. Instead, a new pattern of cFL-evoked activity defined by IOS imaging is found in the peri-infarct rim, extending more anterior, medial, and posterior than the prestroke representation. Notably, compared with controls, the borders between S1HL and S1FL appear blurred 1 month after stroke, because there is significant overlap between the cHL- and cFL-evoked IOS maps (yellow shading). Figure $5 C-E$ shows $\mathrm{Ca}^{2+}$ imaging data from the region sive to cFL somatosensory stimulation (Fig. 6C,D). Limb selectivity was comparable with control S1FL in these shifted cFL regions (neurons 1 and 2), although aberrant responses were still observed (e.g., neuron 3). These posterior-lateral regions were not associated with cFL-evoked $\mathrm{Ca}^{2+}$ activity when imaged in control mice $(n=4)$ (Fig. $6 E-I)$.

As noted previously, $\mathrm{Ca}^{2+}$ field transients provide a measure of local network activity by quantifying the mean somatic, axonal, and dendritic $\left[\mathrm{Ca}^{2+}\right]_{i}$ elevation across the entire image field. Average $\mathrm{Ca}^{2+}$ records representing all animals imaged after targeted S1FL infarction are illustrated in Figure 5, $H$ and $I$ (traces are decimated to $5 \mathrm{~Hz}$; error bars show SEM). Altered limb selectivity is apparent when the poststroke traces in Figure 5, $H$ and $I$, are contrasted with the control averages shown in Figure 3, D and F. For signals recorded in S1HL, increased field activity to stimulation of all limbs is apparent, with the greatest increases in CFL- and iHL-evoked activity peaking 1 month after stroke. In reorganized S1FL, the mean records of field activity are more comparable with control $\mathrm{S} 1 \mathrm{FL}$, although an increase in CHL-evoked field activity is sug- 

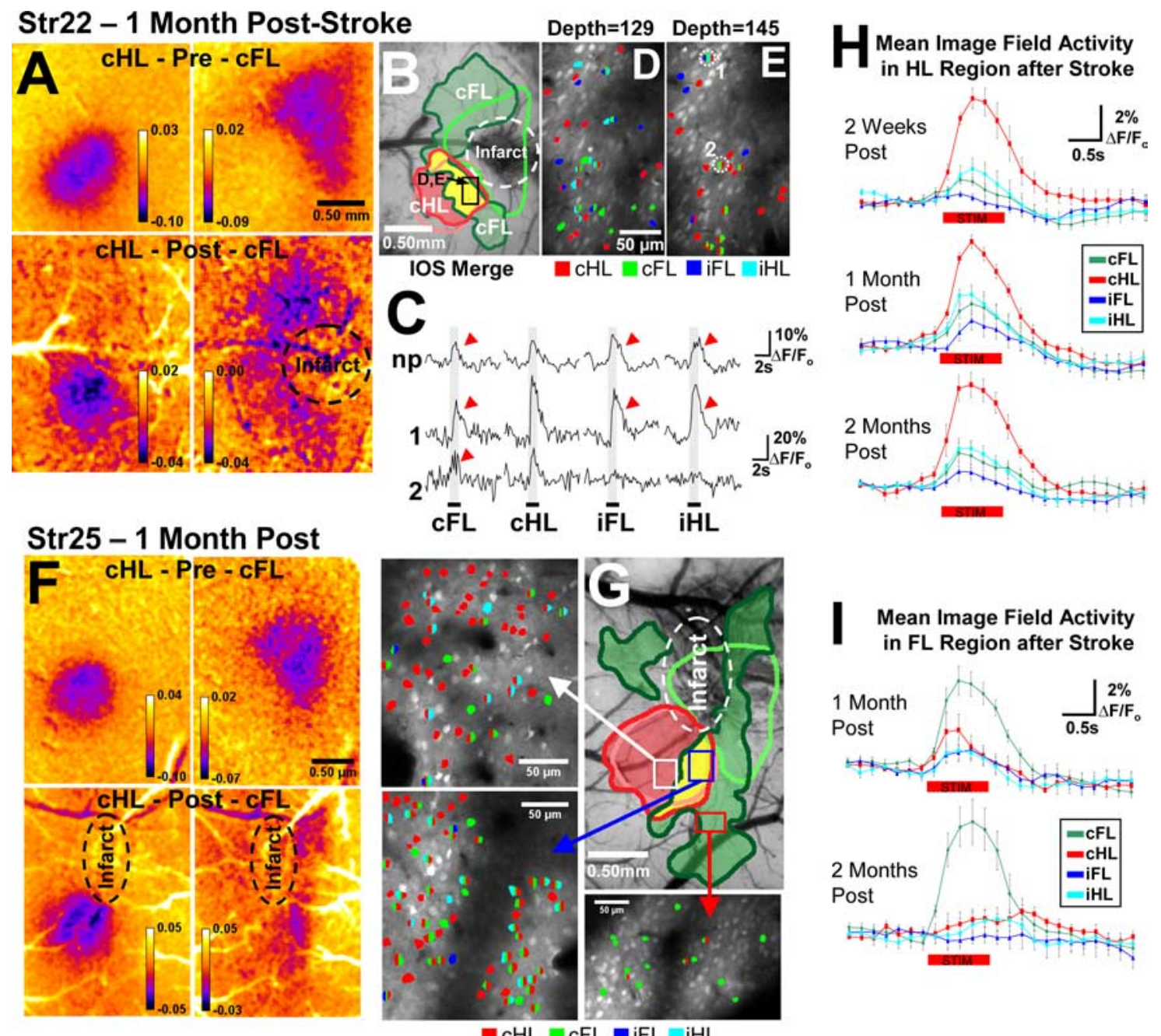

\section{Mean Image Field Activity in FL Region after Stroke}

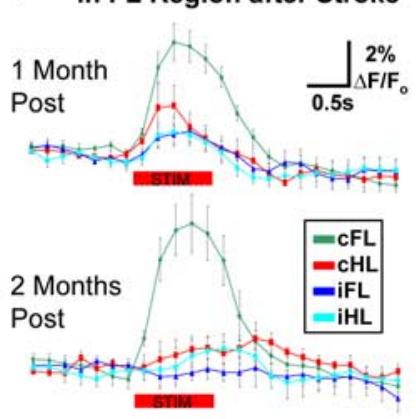

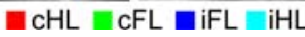

Figure 5. Functional plasticity 1-2 months after stroke. $\boldsymbol{A}, \boldsymbol{B}$, Prestroke and poststroke maps of the sensory-evoked IOSs in mouse Str22, imaged 1 month after targeted photothrombotic infarction of the S1FL. Before stroke, the IOSs evoked by CFL and CHL stimulation had typical topography with a sharp border between the limb representations. After stroke, a new pattern of CFL-evoked IOS activity is observed, whereas CHL-evoked activity is preserved. Considerable overlap exists between $\mathrm{CHL}$ - and CFL-evoked IOS activity. $\mathbf{C}-\boldsymbol{E}$, Representative two-photon $\mathrm{Ca}^{2+}$ imaging data from the region demarcated in $\boldsymbol{B}$. $\mathrm{Ca}^{2+}$ traces (averaged from 10 trials) for two neurons (labeled in $\boldsymbol{E}$ ) and the mean neuropil (np) response (also from the image plane shown in $\boldsymbol{E}$ ) and neuronal response maps (showing above-threshold somatic $\mathrm{Ca}^{2+}$ responses to different limbs, coded by color) at two depths are illustrated. Note the aberrant (nonselective) response to nonpreferred limbs (red arrowheads) in the representative traces, and the reduced limb selectivity in the neuronal response maps. F, G, In mouse Str25, 1 month after stroke, IOS imaging revealed a cFL-evoked pattern of activity with a posterior and medial shift and significant overlap with the $\mathrm{CHL}$-evoked representation. Neuronal response maps in the core of S1HL and in regions of IOS overlap demonstrated reduced limb selectivity (most prominent in regions of IOS overlap). $\mathbf{H}, \mathbf{I}$, Mean $\mathrm{Ca}^{2+}$ records (error bars show the SEM) of the image field responses from all animals for S1HL and S1FL, respectively. These mean traces show that local network activity was altered in both the preserved S1HL and reorganized S1FL, exhibiting reduced limb selectivity (increased amplitude of the image field $\mathrm{Ca}^{2+}$ transient elicited by stimulation of nonpreferred limbs) that peaks 1 month after targeted stroke.

gested at 1 month after stroke. In Figure $6 J$, the mean image field response to cFL stimulation in images collected in regions of the reorganized S1FL found posterior to S1FL and lateral to S1HL (Figs. $5 F-G, 6 A-D$ ) is compared with cFL-evoked activity in the corresponding regions of control animals: enhanced cFL-evoked activity after stroke is apparent.

Multivariate analysis of the average peak $\left[\mathrm{Ca}^{2+}\right]_{\mathrm{i}}$ amplitude of the image field transient elicited by stimulation of each limb during recording in the S1HL of control animals versus animals imaged at 2 weeks, 1 month, or 2 months after stroke revealed a significant effect of time after stroke (Wilk's $\lambda=0.245 ; p=$ 0.006) [supplemental Fig. $3 A$ (left), available at www. jneurosci.org as supplemental material]. Univariate analysis according to limb revealed that peak amplitudes were significantly increased for cFL- (ANOVA, $p=0.037)$ and iHL- $(p<0.001)$ evoked activity. Post hoc analyses using Tukey's HSD suggest that altered iHL-evoked field activity peaks 1 month after stroke (iHL, $p_{\text {Control vs OneMonth }}<0.001 ; \quad p_{\text {Control vs TwoMonths }}=0.007 ;$ $\left.p_{\text {TwoWeeks vs OneMonth }}=0.003 ; p_{\text {OneMonth vs TwoMonths }}=0.019\right)$. Differences in the peak amplitude of the $\mathrm{Ca}^{2+}$ imaging field transients elicited by stimulation of each limb measured in reorganized S1FL [supplemental Fig. 3A (right), available at www. jneurosci.org as supplemental material] did not reach significance when compared with control S1FL (Wilk's $\lambda=$ $0.555 ; p=0.335$ ). However, a multivariate analysis of the peak amplitude of the $\mathrm{Ca}^{2+}$ image field signals focusing on the reorganized S1FL cortex found posterior to S1FL and lateral/posterior to S1HL (where IOSs identified cFL-evoked activity in poststroke mice but not controls) (Figs. $5 F-G, 6 A-D$ ) reveals a significant effect of stroke (Wilk's $\lambda=0.181 ; p=0.02$ ). Specifically, the peak amplitude of the image field transient elicited by cFL stimulation was significantly greater in mice $(n=7)$ imaged $1-2$ months after stroke (ANOVA, $p=0.009$ ) than in naive controls $(n=4)$. Weak image field signals elicited by stimulation of 


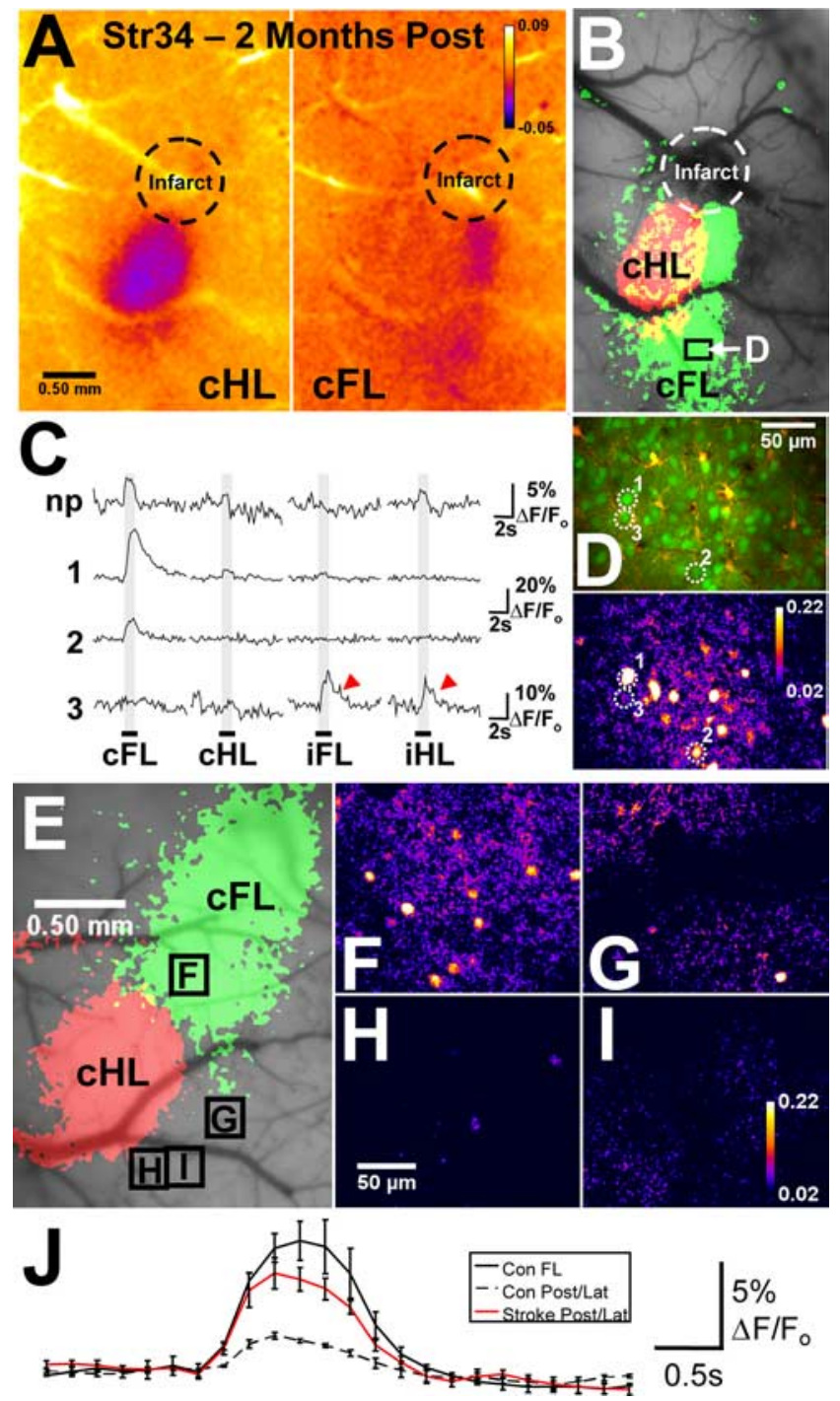

Figure 6. Posterior S1FL reorganization. $\boldsymbol{A}, \boldsymbol{B}$, Mouse $\mathrm{Str} 34$, imaged 2 months after photothrombosis: IOS response maps show increased overlap between the CFL-and CHL-evoked IOSs, and a posterior shift in cFL activation. C, $\mathrm{Ca}^{2+}{ }^{2+}$ imaging (depth, $141 \mu \mathrm{m}$, from the labeled region in the IOS map) demonstrated that the posterior regions of the reorganized cFL representation contained neurons (e.g., 1, 2, labeled in $\boldsymbol{D}$ ) with strong, limb-selective responses to $\mathrm{CFL}$ stimulation. Aberrant responses (red arrowheads) were observed in some neurons (e.g., 3). D, A difference image illustrating the strong $\mathrm{Ca}^{2+}$-induced fluorescence signal $\left(\Delta F / F_{0}\right)$ over $\sim 3 \mathrm{~s}$ after $\mathrm{CFL}$ stimulation onset in the image section from which the representative neurons in $C$ were selected [shown below the corresponding 2-photon image of OGB-1 (green) and SR101 (red) labeling]. E, IOS maps from a naive animal showing the $\mathrm{CLL}$ - and $\mathrm{CHL}$-evoked representations. $\boldsymbol{F}-\mathrm{I}$, Difference images illustrating the $\Delta F / F_{0}$ in $\mathrm{S} 1 \mathrm{FL}$ and regions of the cortex posterior to S1FL and lateral/posterior to S1HL during CFL stimulation (over $\sim 3$ s after stimulus onset) from the mouse shown in $\boldsymbol{E}$ (borders of the image sections denoted on the IOS map in $\boldsymbol{E}$ ). Importantly, $\mathrm{CFL}$ stimulation evoked little or no $\mathrm{Ca}^{2+}$ signal in the posterior and lateral regions in control animals.J, Mean records of the $\mathrm{Ca}^{2+}$ image field activity elicited by cFL stimulation in the S1FL $(n=$ $2^{*}$ ) and posterior/lateral (Post/Lat) cortex $(n=4)$ of control mice and the same regions in animals imaged $1-2$ months after stroke $(n=7)$. The cFL-evoked activity revealed by two-photon $\mathrm{Ca}^{2+}$ imaging in these posterior and lateral regions was significantly stronger in stroke animals compared with controls (ANOVA, $p=0.009$ ), confirming the posterior S1FL reorganization suggested by the IOS maps. *In two mice, OGB-1 AM was microinjected in S1FL and the control regions posterior and lateral to S1FL/S1HL, whereas it was injected into S1HL and the control regions in two other controls.

the other limbs did not significantly differ between stroke and naive animals in these regions.

Although field activity is useful for assessing and quantifying altered limb selectivity after stroke, the strength of two-photon $\mathrm{Ca}^{2+}$ imaging lies in its single-cell resolution and ability to separate somatic and neuropil activity. To that end, we quantified neuronal somatosensory response properties in 5667 layer 2/3 neurons (S1HL: 2 weeks after stroke $=1300$ neurons, 4 mice; 1 month after stroke $=1240$ neuron, 6 mice; 2 months after stroke $=1163$ neurons, 6 mice; S1FL: 1 month after stroke $=1174$ neurons, 6 mice; 2 months after stroke $=790$ neurons, 4 mice) and the surrounding neuropil after targeted S1FL stroke. Figure $7 \mathrm{~A}$ (left) shows the percentage of neurons with above-threshold responses to stimulation of each of the limbs in S1HL in controls and at 2 weeks, 1 month, and 2 months after stroke. Strikingly, neurons in the S1HL of all mice imaged at 1 month after stroke (6/6) showed altered limb selectivity (prevalence of above-threshold aberrant responses $>2$ SD above the mean prevalence in control S1HL), and altered limb selectivity persisted in S1HL in 50\% (3/6) of mice imaged 2 months after stroke. The most prominent changes were found at 1 month after stroke, when the prevalence of cFL-, iHL-, and iFL-evoked responses (within S1HL) was $\sim 10$-fold greater than controls. Multivariate analysis confirmed a significant effect of targeted S1FL stroke on limb selectivity in S1HL (Wilk's $\lambda=0.220 ; p=0.003$ ), and univariate analyses determined that the prevalence of above-threshold responses to stimulation of each limb was altered (cHL, $p=0.045$; cFL, $p=0.007$; iFL, $p<$ $0.001 ; \mathrm{iHL}, p=0.015)$. Significant post hoc results (Tukey's HSD) again highlighted that altered limb selectivity peaked 1 month after targeted ischemic insult (cHL: $p_{\text {Control vs OneMonth }}=0.029$; cFL: $\quad p_{\text {Control vs OneMonth }}=0.006$, $p_{\text {TwoWeeks }}$ vs OneMonth $=0.029 ;$ iFL: P Control vs OneMonth $\quad<\quad 0.001$ $p_{\text {TwoWeeks vs OneMonth }}=0.001$, POneMonth vs TwoMonths $=0.004 ;$ iHL: $p_{\text {Control vs OneMonth }}=0.012$ ). Additionally, whereas aberrant responses in the S1HL of control animals and mice 2 weeks after stroke had a trend toward reduced peak $\Delta F / F_{\mathrm{o}}$ amplitudes (relative to $\mathrm{CHL}$-evoked responses), the peak amplitudes of aberrant responses at 1 and 2 months after stroke were equivalent to or greater than cHL-evoked responses (Table 2). Neither the decay constant nor the time to peak from stimulus onset of S1HL neurons exhibiting above-threshold responses to $\mathrm{cHL}$ stimulation were altered by ischemic insult (Tables 2, 3). Relative to controls, the prevalence of neurons in S1HL stimulated by movement of more than one limb was greatly increased. In control S1HL, an average of $28.2 \%$ of neurons respond to stimulation of a single limb, $1.3 \%$ to stimulation of two limbs (individually), and $0.1 \%$ to stimulation of three or more limbs. In contrast, at 1 month after stroke, the percentage of neurons with above-threshold responses to one, two, or three or more limbs was $45.2,14.8$, and $5.2 \%$, respectively. Averaging all poststroke time points, the prevalence of above-threshold responses to one, two, or three or more limbs was 144, 591, and $1620 \%$ of controls, 
respectively. Altered-limb selectivity was also apparent in nonsomatic $\mathrm{Ca}^{2+}$ transients in the neuropil in S1HL. Whereas above-threshold transients in response to stimulation of the nonpreferred limbs were rare in the neuropil of control animals, 1 month after stroke $\sim 70 \%$ of image planes tested exhibited above-threshold responses to iHL stimulation, and $\sim 50 \%$ exhibited nonsomatic activation after $\mathrm{cFL}$ stimulation (Table 4). At 2 months after stroke, 20-25\% of image planes demonstrated above-threshold responses to iHL and CFL stimulation.

As evidenced by Figure 5, $B-E$ and $G$, $\mathrm{Ca}^{2+}$ imaging suggested that functional rewiring, manifested in S1HL and S1FL as altered limb selectivity in neuronal somata, is enhanced in the border regions of S1HL and S1FL, where overlap between the cFL- and cHL-evoked IOSs is observed after stroke. To further examine, this we compared the limb selectivity of neurons in the core of S1HL (nonoverlapping regions of the IOS response maps) to the limb selectivity of neurons in regions of S1HL/S1FL overlap in animals imaged 1 or 2 months after stroke (Fig. $7 A$, right column). Multivariate analysis confirmed that altered limb selectivity is more pronounced in regions of S1HL/S1FL overlap (Wilk's $\lambda=0.117 ; p<0.001$ ), with a significant increase in $\mathrm{cFL}$-evoked neuronal responses (ANOVA, $p=0.044$ ).

The prevalence of above-threshold responses to limb stimulation in S1FL of control and stroke animals is shown in Figure $7 B$ (left). Altered limb selectivity in neurons within the reorganized S1FL representation was observed in $83 \%(5 / 6)$ of mice imaged at 1 month after stroke and 50\% (2/4) of mice imaged at 2 months after ischemic insult. Multivariate analysis suggested a weak effect of time after stroke on limb selectivity in the reorganized S1FL (Wilk's $\lambda=0.367 ; p=0.051$ ). Univariate tests reveal that cHLevoked responses are increased in S1FL after stroke $(p=0.043)$, and post hoc comparisons confirm that this effect is greatest 1 month after stroke (Tukey's HSD, $p_{\text {Control vs OneMonth }}=0.036$ ). After stroke (averaging 1 and 2 month poststroke data), the prevalence of above-threshold responses to cHL stimulation alone in S1FL was $609 \%$ that of controls, and the prevalence of neurons responding to both $\mathrm{cHL}$ and cFL stimulation was $1396 \%$ of control levels. Overall, the prevalence of neurons responsive to only single limbs in reorganized S1FL was $158 \%$ of controls, whereas responses to two limbs or three or more limbs were 556 and $601 \%$ of control levels, respectively. The kinetics of the sensory-evoked somatic responses in reorganized S1FL were comparable with controls (Tables 1, 3), and there was no significant difference in limb selectivity between anterior (primarily preserved tissue proximal to the stroke) or posterior (reorganized) S1FL regions after stroke (Fig. $7 B$, right). Nonsomatic activity in reorganized S1FL showed a similar pattern of moderately altered sensoryevoked activity after stroke (Table 5).

The mean sensory-evoked $\mathrm{Ca}^{2+}$ transients in neuronal somata and the neuropil in S1HL and S1FL of all control animals and at all time points studied after stroke are shown in supple- mental Figure 3, $B$ and $C$ (available at www.jneurosci.org as supplemental material). These averaged records represent the mean $\left[\mathrm{Ca}^{2+}\right]_{\mathrm{i}}$ signal of all neurons or regions of the neuropil in one image plane per animal (i.e., includes neurons both above and below threshold criteria). Altered limb selectivity in S1HL, particularly in response to cFL and iHL stimulation, is apparent after stroke in both neuronal somata and the neuropil, whereas the mean transients in reorganized S1FL are moderately altered but more comparable with control S1FL. Although the mean somatic and neuropil transients correlate well, subtle differences are apparent: notably, cFL-evoked activity in S1HL was enhanced relative to controls in the neuropil at 2 weeks after stroke, but not in neuronal somata (cFL-evoked responses were enhanced in both the neuropil and neuronal somata at 1 month after stroke). Importantly, we reach similar conclusions by analysis of thresholded neuronal responses, mean somatic and neuropil transients (including above- and below-threshold records), and image field data (pooled somatic, axonal, and dendritic transients), suggesting that our procedure for thresholding signals was not leading to erroneous identification of altered limb selectivity and neurons that responded to multiple limbs.

\section{Sensory-evoked astrocyte $\mathrm{Ca}^{2+}$ signals}

Previous two-photon $\mathrm{Ca}^{2+}$ imaging studies (Wang et al., 2006; Winship et al., 2007) have revealed sensory-evoked $\mathrm{Ca}^{2+}$ signals in astrocyte somata and endfeet in layer $2 / 3$ of the somatosensory cortex. To examine whether the prevalence of these responses was altered in peri-infarct cortex, we examined sensory-evoked activity of 1437 astrocyte somata in control (507 astrocytes, 12 mice) and poststroke (930 astrocytes, 18 mice) animals. Figure $8 \mathrm{~A}$ 


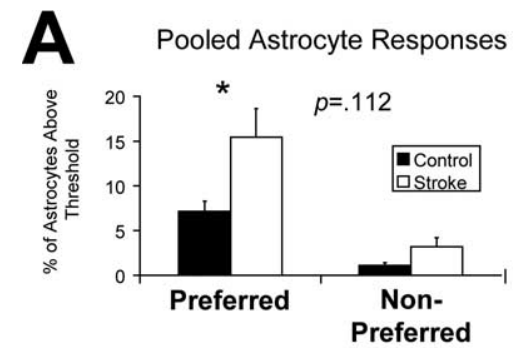

B

Astrocyte Responses in HL Region

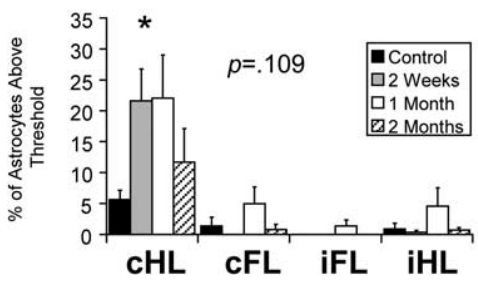

C HL Astrocyte Responses in IOS Overlap

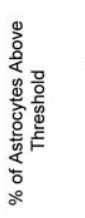

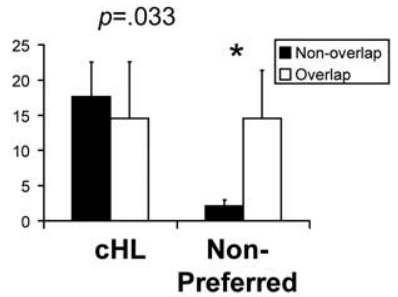

D

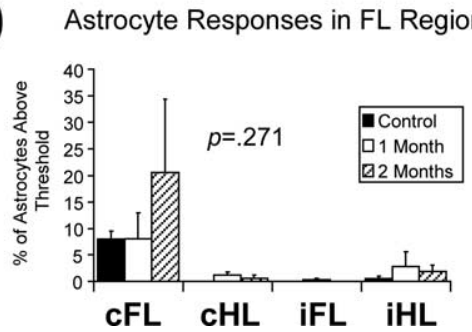

Figure 8. Astrocyte signaling after stroke. $A$, Pooled data examining above-threshold $\mathrm{Ca}^{2+}$ responses from all astrocytes imaged in S1FL and S1HL suggested a weak effect of stroke on astrocyte responses to preferred and nonpreferred limbs ( $p=$ 0.112). Univariate analysis confirmed that sensory-evoked $\mathrm{Ca}^{2+}$ signals in response to stimulation of the preferred limb (cFL and CHL for S1FL and S1HL, respectively) were more common after stroke. $B$, In S1HL, above-threshold responses to CHL stimulation were more common after stroke (ANOVA, $p=0.043$ ), although the overall effect on limb selectivity was relatively weak (MANOVA, $p=0.109$ ). C, As was the case for neuronal somata, limb selectivity of astrocytes differed in the S1HL core regions compared with regions of overlap between the $\mathrm{CFL}$ - and $\mathrm{CHL}$-evoked intrinsic signal (MANOVA, $p=0.033$ ), because above-threshold responses to the nonpreferred (aberrant) limbs were more common in areas of overlap $(p=0.031)$. $\boldsymbol{D}$, The prevalence of sensory-evoked $\mathrm{Ca}^{2+}$ signals in response to stimulation of the different limbs was not significantly altered in S1FL (MANOVA, $p=0.271) .{ }^{*} p<0.05$.

shows the prevalence of above-threshold $\mathrm{Ca}^{2+}$ responses to stimulation of the preferred (cFL in S1FL or cHL in S1HL) or nonpreferred limbs. Overall, a trend toward increased astrocyte responses to both preferred and nonpreferred limbs after stroke was suggested, but did not reach significance in multivariate analysis (Wilk's $\lambda=0.850 ; p=0.112$ ). However, univariate analysis revealed that there was a significant increase in the prevalence of responses to preferred limb stimulation in primary somatosensory cortex $($ control $=7.1 \pm 1.1 \%$; stroke $=15.4 \pm 3.2 \%$; $p=$ $0.048)$. Independent multivariate analyses of astrocyte responses in S1HL and S1FL (Fig. $8 B, D$, respectively) suggests an increase in cHL-responsive astrocytes after stroke in S1HL (MANOVA, Wilk's $\lambda=0.400 ; p=0.109$; ANOVA on cHL prevalence, $p=$ $0.043)$, but no significant change sensory-evoked astrocyte activity in reorganized or preserved S1FL (Wilk's $\lambda=0.526$; $p=$ 0.271 ). Additionally, the selectivity of astrocyte responses between regions of overlap between CHL- and cFL-evoked IOSs and regions without IOS overlap (Fig. $8 \mathrm{C}$ ) was significantly different (MANOVA, Wilk's $\lambda=0.539 ; p=0.033$ ), largely because of a significant increase in above-threshold responses to nonpreferred limbs in regions of overlap (ANOVA, $p=0.031$ ). These findings suggest that mechanisms for rapid neuron-astrocyte mechanisms for communication are preserved, or even enhanced, after stroke.

\section{Discussion}

Comparison with previous studies of plasticity after stroke

In patients (Rossini et al., 2007) and animal stroke models (Abo et al., 2001; Dijkhuizen et al., 2001, 2003; Wei et al., 2001; Zepeda et al., 2003, 2004), ipsilesional regional reorganization as observed here is correlated with improved behavioral recovery. In rats with small infarcts, activity within the stroke-affected cortex was required for reaching task performance (Biernaskie et al.,
2005). Additionally, structural plasticity is enhanced in perilesional neurons (Stroemer et al., 1995; Gonzalez and Kolb, 2003; Brown et al., 2007), and peri-infarct cortex is hyperexcitable after stroke (BuchkremerRatzmann et al., 1996; Schiene et al., 1996; Mittmann et al., 1998; Neumann-Haefelin et al., 1998, 1999; Redecker et al., 2002; Carmichael, 2003; Centonze et al., 2007), creating an environment suitable for functional rewiring. Enlarged receptive fields in neurons in somatosensory (Jenkins and Merzenich, 1987; Reinecke et al., 2003) cortices after focal lesions were suggested by extracellular recordings during sensory stimulation. However, these previous studies did not examine sensory-evoked responses to stimulation of other (nonpreferred) limbs, nor can they replicate the high spatial resolution and unambiguous identification of large numbers of neurons provided by $\mathrm{Ca}^{2+}$ imaging.

\section{Models for functional rewiring}

Two-photon $\mathrm{Ca}^{2+}$ imaging detects $\left[\mathrm{Ca}^{2+}\right]_{\mathrm{i}}$ elevations primarily because of action potentials in neuronal somata (Stosiek et al., 2003; Berger et al., 2007; Sato et al., 2007). Previous electrophysiological studies demonstrated tightly tuned suprathreshold receptive fields in somatosensory cortex (Simons, 1978; Moore and Nelson, 1998; Zhu and Connors, 1999; Petersen and Diamond, 2000; Brecht et al., 2003; Wilent and Contreras, 2005; Berger et al., 2007). Conversely, subthreshold changes in membrane potential are not dependent on spiking (Berger et al., 2007), and have broadly tuned receptive fields. It is conceivable that layer $2 / 3$ neurons normally receive subthreshold synaptic inputs from all four limbs (Ferezou et al., 2007; Petersen, 2007). After stroke, somatosensory cortex may be rewired, such that previously subthreshold activity causes action potential firing, inducing broader receptive field tuning (reduced limb selectivity).

Regarding mechanism, long-lasting neuronal hyperexcitability in peri-infarct cortex is observed after stroke (Domann et al., 1993; Buchkremer-Ratzmann et al., 1996; Schiene et al., 1996; Mittmann et al., 1998; Neumann-Haefelin et al., 1998, 1999; Redecker et al., 2002), peaking 4 weeks after stroke and persisting for $60 \mathrm{~d}$ (Mittmann et al., 1998). Zepeda et al. (2004) demonstrated that regional reorganization 5 weeks after photothrombosis correlates with an increase in NMDA-receptor subunit levels from 1-5 weeks after stroke, while GABA levels remain reduced. Anatomically, axonal sprouting in peri-infarct cortex is significantly enhanced for 2 weeks after ischemia ( $\mathrm{Ng}$ et al., 1988; Stroemer et al., 1995; Li et al., 1998; Carmichael and Chesselet, 2002). Enhanced synaptogenesis is supported by in vivo studies of structural plasticity (Brown et al., 2007), demonstrating that spine formation on the apical dendrites of layer 5 neurons is enhanced in peri-infarct cortex for 6 weeks or more. Local and distal intracortical projection patterns are also altered by ischemia (Carmichael et al., 2001; Dancause et al., 2005). Lesion studies in visual cortex suggest that plasticity is intrinsic to the cortex, and reorganization of thalamocortical afferents cannot account for cortical reorganization (Gilbert and Wiesel, 1992; Darian-Smith 
and Gilbert, 1994; Gilbert, 1998). However, although focal stroke has been shown to reduce thalamocortical projections to somatosensory cortex (Carmichael et al., 2001), preserved thalamocortical afferents may provide input to more neurons in reorganized S1FL because of axonal sprouting and hyperexcitability of periinfarct cortex. In vivo imaging of axonal sprouting (PorteraCailliau et al., 2005) could be used to assess the plasticity and nature of new inputs to peri-infarct cortex. Importantly, the time course of these molecular and anatomical alterations correlates well with the temporal profile of the functional rewiring we observed in layer $2 / 3$ somatosensory neurons after stroke.

Axonal sprouting, synaptogenesis, and hyperexcitability may facilitate the transition of S1HL/S1FL border regions from functionally segregated areas where neurons respond only to stimulation of the preferred limb into less selective regions where neurons process multiple sensory streams. New synapses formed via sprouting of regenerating intracortical axons from surviving neurons in peri-infarct primary somatosensory cortex, ipsilesional secondary somatosensory areas, or contralateral primary somatosensory areas would facilitate functional rewiring in layer $2 / 3$. Reduced inhibition and increased glutamate transmission peaking 1 month after stroke could strengthen neurotransmission at nascent synapses through activity-dependent plasticity. Previously subthreshold postsynaptic potentials elicited by stimulation of the nonpreferred limbs might be potentiated by cortical hyperexcitability, giving rise to more broadly tuned suprathreshold receptive fields (reduced limb selectivity). After longer recoveries, neurons become more selective for cFL stimulation alone, producing more tightly tuned receptive fields in neurons within reorganized limb representations.

\section{Is rewiring of a subset of neurons significant?}

Recent studies demonstrate that small subsets of somatosensory neurons are capable of influencing animal behavior. Huber et al. (2008) introduced light-gated channels, capable of generating action potentials after brief photostimulation, into small subsets of layer $2 / 3$ neurons in the barrel cortex of mice and demonstrated that eliciting action potentials in 60-300 neurons was sufficient to influence perceptual decisions. Similarly, Houweling and Brecht (2008) demonstrated that stimulation of single somatosensory neurons affected performance in a detection task. These results suggest that the neural code for somatosensation is sparse, and small subsets of neurons may have considerable impact on behavior. Our data suggest that $\sim 10-30 \%$ of all neurons in S1FL and S1HL responded to nonpreferred limbs 1-2 months after stroke (a 400-1200\% increase from controls). Given models suggesting sparse somatosensory coding, this subset of functionally rewired neurons may have considerable influence on behavior during recovery. Analysis of behavioral recovery in our laboratory (C. E. Brown, K. Aminoltejari, H. Erb, I. R. Winship, and T. H. Murphy, unpublished results) suggests that use of the impaired limb is reduced 2 weeks after targeted S1FL stroke, but recovers significantly after $3-8$ weeks, correlating with the time course of plasticity observed here.

\section{Why is plasticity enhanced in border regions?}

Enhanced functional plasticity near the S1FL/S1HL border might be predicted based on several lines of evidence. Photothrombotic strokes targeted the center of S1FL, such that some neurons in the medialmost or posteriormost extents of S1FL survive the initial insult, but lack some of their preferred inputs, perhaps rendering them more responsive to other signals. Axonal sprouting and synaptogenesis by surviving neurons could account for a poste- rior and medial expansion in the S1FL representation. Commissural connections with homotopic cortex in the opposite hemisphere are reported in sensorimotor cortex (Donoghue and Parham, 1983), and sprouting of commissural axons in S1HL might account for neurons with responses to iHL stimulation. It is also conceivable that neurons at borders receive mixed inputs and are therefore more malleable (Dragoi et al., 2000, 2001; Schummers et al., 2002, 2004).

Two-photon $\mathrm{Ca}^{2+}$ imaging has previously demonstrated that neurons in layer $2 / 3$ of the cat visual cortex are organized into discrete orientation columns, with borders 1-2 somata wide (Ohki et al., 2005, 2006). The sharp borders defined by somatic activity exist on a finer spatial scale than their dendritic arbors, which overlap with neighboring neurons. Functional segregation, without anatomical separation, may result from selective connections between neurons with similar tuning, or uneven weighting of synaptic strength on either side of the border. After stroke, functional segregation might be reduced by axonal sprouting, synaptogenesis, and neuronal hyperexcitability, reducing limb selectivity and blurring functional borders. As such, subthreshold activation may extend beyond the borders defined by imaging of somatic $\mathrm{Ca}^{2+}$ transients in control animals. After stroke, neuronal hyperexcitability may potentiate subthreshold activity, expanding the suprathreshold S1FL representation and reducing limb selectivity at the S1HL/S1FL border.

\section{Functional consequences of S1FL reorganization}

Using regional and single-cell in vivo imaging, we show that surviving neurons are remarkably plastic and can process multiple streams of sensory information after stroke. However, this wider repertoire of neuronal processing does not preclude neurons from performing their normal duties, and over time neurons within the heart of a reorganized territory exhibit a preferred stimulus. A remaining question is how altered limb selectivity aids recovery. It is conceivable that neurons responding to multiple limbs reflect a transitory phase from involvement in one sensorimotor function to a new function (replacing processing lost because of stroke). Inputs from seemingly irrelevant limbs (relative to the area damaged) may also provide background activity (Waters and Helmchen, 2006) useful during plasticity in reorganized representations, or be a byproduct of extensive axonal sprouting and hyperexcitability (Carmichael, 2003). If reduced selectivity near the S1FL/S1HL border reflects a transition from predominantly hindlimb-associated sensation to forelimb sensorimotor function, regions of the somatosensory cortex posterior to S1FL and lateral to S1HL associated with shoulder/neck and trunk sensation (Paxinos and Franklin, 2001) may exhibit similar altered selectivity after targeted S1FL stroke. Although we did not assess sensory-evoked responses to these regions, one might predict the same pattern of reduced stimulus selectivity as S1FL reorganization proceeds 1-2 months after stroke. Thus, it is possible that reorganization of S1FL may facilitate recovery of forelimb function at some expense to hindlimb or trunk somatosensation. Although the functional consequence of altered limb selectivity within S1FL and nearby regions is unknown, it nonetheless provides important mechanistic clues into the process by which remapping occurs in single neurons.

\section{References}

Abo M, Chen Z, Lai LJ, Reese T, Bjelke B (2001) Functional recovery after brain lesion-contralateral neuromodulation: an fMRI study. NeuroReport 12:1543-1547.

Berger T, Borgdorff A, Crochet S, Neubauer FB, Lefort S, Fauvet B, Ferezou I, 
Carleton A, Lüscher HR, Petersen CC (2007) Combined voltage and calcium epifluorescence imaging in vitro and in vivo reveals subthreshold and suprathreshold dynamics of mouse barrel cortex. J Neurophysiol 97:3751-3762.

Biernaskie J, Corbett D (2001) Enriched rehabilitative training promotes improved forelimb motor function and enhanced dendritic growth after focal ischemic injury. J Neurosci 21:5272-5280.

Biernaskie J, Szymanska A, Windle V, Corbett D (2005) Bi-hemispheric contribution to functional motor recovery of the affected forelimb following focal ischemic brain injury in rats. Eur J Neurosci 21:989-999.

Bonhoeffer T, Grinvald A (1996) Optical imaging based on intrinsic signals: the methodology. In: Brain mapping: the methods (Toga A, Mazziotta J, eds). New York: Academic.

Brecht M, Roth A, Sakmann B (2003) Dynamic receptive fields of reconstructed pyramidal cells in layers 3 and 2 of rat somatosensory barrel cortex. J Physiol (Lond) 553:243-265.

Brown CE, Li P, Boyd JD, Delaney KR, Murphy TH (2007) Extensive turnover of dendritic spines and vascular remodeling in cortical tissues recovering from stroke. J Neurosci 27:4101-4109.

Brown CE, Wong C, Murphy TH (2008) Rapid morphological plasticity of peri-infarct dendritic spines after focal ischemic stroke. Stroke 39:1286-1291.

Buchkremer-Ratzmann I, August M, Hagemann G, Witte OW (1996) Electrophysiological transcortical diaschisis after cortical photothrombosis in rat brain. Stroke 27:1105-1109; discussion 1109-1011.

Carmichael ST (2003) Plasticity of cortical projections after stroke. Neuroscientist 9:64-75.

Carmichael ST (2006) Cellular and molecular mechanisms of neural repair after stroke: making waves. Ann Neurol 59:735-742.

Carmichael ST, Chesselet MF (2002) Synchronous neuronal activity is a signal for axonal sprouting after cortical lesions in the adult. J Neurosci 22:6062-6070.

Carmichael ST, Wei L, Rovainen CM, Woolsey TA (2001) New patterns of intracortical projections after focal cortical stroke. Neurobiol Dis 8:910-922.

Castro-Alamancos MA, Borrel J (1995) Functional recovery of forelimb response capacity after forelimb primary motor cortex damage in the rat is due to the reorganization of adjacent areas of cortex. Neuroscience 68:793-805.

Centonze D, Rossi S, Tortiglione A, Picconi B, Prosperetti C, De Chiara V, Bernardi G, Calabresi P (2007) Synaptic plasticity during recovery from permanent occlusion of the middle cerebral artery. Neurobiol Dis 27:44-53.

Chollet F, DiPiero V, Wise RJ, Brooks DJ, Dolan RJ, Frackowiak RS (1991) The functional anatomy of motor recovery after stroke in humans: a study with positron emission tomography. Ann Neurol 29:63-71.

Cramer SC, Chopp M (2000) Recovery recapitulates ontogeny. Trends Neurosci 23:265-271.

Dancause N, Barbay S, Frost SB, Plautz EJ, Chen D, Zoubina EV, Stowe AM, Nudo RJ (2005) Extensive cortical rewiring after brain injury. J Neurosci 25:10167-10179.

Darian-Smith C, Gilbert CD (1994) Axonal sprouting accompanies functional reorganization in adult cat striate cortex. Nature 368:737-740.

Devor A, Dunn AK, Andermann ML, Ulbert I, Boas DA, Dale AM (2003) Coupling of total hemoglobin concentration, oxygenation, and neural activity in rat somatosensory cortex. Neuron 39:353-359.

Dijkhuizen RM, Ren J, Mandeville JB, Wu O, Ozdag FM, Moskowitz MA, Rosen BR, Finklestein SP (2001) Functional magnetic resonance imaging of reorganization in rat brain after stroke. Proc Natl Acad Sci USA 98:12766-12771.

Dijkhuizen RM, Singhal AB, Mandeville JB, Wu O, Halpern EF, Finklestein SP, Rosen BR, Lo EH (2003) Correlation between brain reorganization, ischemic damage, and neurologic status after transient focal cerebral ischemia in rats: a functional magnetic resonance imaging study. J Neurosci 23:510-517.

Domann R, Hagemann G, Kraemer M, Freund HJ, Witte OW (1993) Electrophysiological changes in the surrounding brain tissue of photochemically induced cortical infarcts in the rat. Neurosci Lett 155:69-72.

Dombeck DA, Khabbaz AN, Collman F, Adelman TL, Tank DW (2007) Imaging large-scale neural activity with cellular resolution in awake, mobile mice. Neuron 56:43-57.
Donoghue JP, Parham C (1983) Afferent connections of the lateral agranular field of the rat motor cortex. J Comp Neurol 217:390-404.

Dragoi V, Sharma J, Sur M (2000) Adaptation-induced plasticity of orientation tuning in adult visual cortex. Neuron 28:287-298.

Dragoi V, Rivadulla C, Sur M (2001) Foci of orientation plasticity in visual cortex. Nature 411:80-86.

Enright LE, Zhang S, Murphy TH (2007) Fine mapping of the spatial relationship between acute ischemia and dendritic structure indicates selective vulnerability of layer $\mathrm{V}$ neuron dendritic tufts within single neurons in vivo. J Cereb Blood Flow Metab 27:1185-1200.

Ferezou I, Haiss F, Gentet LJ, Aronoff R, Weber B, Petersen CC (2007) Spatiotemporal dynamics of cortical sensorimotor integration in behaving mice. Neuron 56:907-923.

Fridman EA, Hanakawa T, Chung M, Hummel F, Leiguarda RC, Cohen LG (2004) Reorganization of the human ipsilesional premotor cortex after stroke. Brain 127:747-758.

Friel KM, Heddings AA, Nudo RJ (2000) Effects of postlesion experience on behavioral recovery and neurophysiologic reorganization after cortical injury in primates. Neurorehabil Neural Repair 14:187-198.

Frost SB, Barbay S, Friel KM, Plautz EJ, Nudo RJ (2003) Reorganization of remote cortical regions after ischemic brain injury: a potential substrate for stroke recovery. J Neurophysiol 89:3205-3214.

Frostig RD, Lieke EE, Ts'o DY, Grinvald A (1990) Cortical functional architecture and local coupling between neuronal activity and the microcirculation revealed by in vivo high-resolution optical imaging of intrinsic signals. Proc Natl Acad Sci USA 87:6082-6086.

Garaschuk O, Milos RI, Konnerth A (2006) Targeted bulk-loading of fluorescent indicators for two-photon brain imaging in vivo. Nat Protoc 1:380-386.

Gharbawie OA, Gonzalez CL, Williams PT, Kleim JA, Whishaw IQ (2005) Middle cerebral artery (MCA) stroke produces dysfunction in adjacent motor cortex as detected by intracortical microstimulation in rats. Neuroscience 130:601-610.

Gilbert CD (1998) Adult cortical dynamics. Physiol Rev 78:467-485.

Gilbert CD, Wiesel TN (1992) Receptive field dynamics in adult primary visual cortex. Nature 356:150-152.

Gonzalez CL, Kolb B (2003) A comparison of different models of stroke on behaviour and brain morphology. Eur J Neurosci 18:1950-1962.

Grinvald A, Lieke E, Frostig RD, Gilbert CD, Wiesel TN (1986) Functional architecture of cortex revealed by optical imaging of intrinsic signals. Nature 324:361-364.

Houweling AR, Brecht M (2008) Behavioural report of single neuron stimulation in somatosensory cortex. Nature 451:65-68.

Huber D, Petreanu L, Ghitani N, Ranade S, Hromádka T, Mainen Z, Svoboda K (2008) Sparse optical microstimulation in barrel cortex drives learned behaviour in freely moving mice. Nature 451:61-64.

Jaillard A, Martin CD, Garambois K, Lebas JF, Hommel M (2005) Vicarious function within the human primary motor cortex? A longitudinal fMRI stroke study. Brain 128:1122-1138.

Jenkins WM, Merzenich MM (1987) Reorganization of neocortical representations after brain injury: a neurophysiological model of the bases of recovery from stroke. Prog Brain Res 71:249-266.

Kerr JN, Greenberg D, Helmchen F (2005) Imaging input and output of neocortical networks in vivo. Proc Natl Acad Sci USA 102:14063-14068.

Kerr JN, de Kock CP, Greenberg DS, Bruno RM, Sakmann B, Helmchen F (2007) Spatial organization of neuronal population responses in layer 2/3 of rat barrel cortex. J Neurosci 27:13316-13328.

Kleim JA, Bruneau R, VandenBerg P, MacDonald E, Mulrooney R, Pocock D (2003) Motor cortex stimulation enhances motor recovery and reduces peri-infarct dysfunction following ischemic insult. Neurol Res 25:789-793.

Li Y, Jiang N, Powers C, Chopp M (1998) Neuronal damage and plasticity identified by microtubule-associated protein 2 , growth-associated protein 43 , and cyclin D1 immunoreactivity after focal cerebral ischemia in rats. Stroke 29:1972-1980; discussion 1980-1981.

Mao BQ, Hamzei-Sichani F, Aronov D, Froemke RC, Yuste R (2001) Dynamics of spontaneous activity in neocortical slices. Neuron 32:883-898.

Mittmann T, Qü M, Zilles K, Luhmann HJ (1998) Long-term cellular dysfunction after focal cerebral ischemia: In vitro analyses. Neuroscience $85: 15-27$

Moore CI, Nelson SB (1998) Spatio-temporal subthreshold receptive fields 
in the vibrissa representation of rat primary somatosensory cortex. J Neurophysiol 80:2882-2892.

Mrsic-Flogel TD, Hofer SB, Ohki K, Reid RC, Bonhoeffer T, Hübener M (2007) Homeostatic regulation of eye-specific responses in visual cortex during ocular dominance plasticity. Neuron 54:961-972.

Neumann-Haefelin T, Staiger JF, Redecker C, Zilles K, Fritschy JM, Möhler H, Witte OW (1998) Immunohistochemical evidence for dysregulation of the GABAergic system ipsilateral to photochemically induced cortical infarcts in rats. Neuroscience 87:871-879.

Neumann-Haefelin T, Bosse F, Redecker C, Müller HW, Witte OW (1999) Upregulation of GABAA-receptor alpha1- and alpha2-subunit mRNAs following ischemic cortical lesions in rats. Brain Res 816:234-237.

Ng SC, de la Monte SM, Conboy GL, Karns LR, Fishman MC (1988) Cloning of human GAP-43: growth association and ischemic resurgence. Neuron 1:133-139.

Nimmerjahn A, Kirchhoff F, Kerr JN, Helmchen F (2004) Sulforhodamine 101 as a specific marker of astroglia in the neocortex in vivo. Nat Methods $1: 31-37$.

Nudo RJ, Milliken GW (1996) Reorganization of movement representations in primary motor cortex following focal ischemic infarcts in adult squirrel monkeys. J Neurophysiol 75:2144-2149.

Ohki K, Chung S, Ch'ng YH, Kara P, Reid RC (2005) Functional imaging with cellular resolution reveals precise micro-architecture in visual cortex. Nature 433:597-603.

Ohki K, Chung S, Kara P, Hübener M, Bonhoeffer T, Reid RC (2006) Highly ordered arrangement of single neurons in orientation pinwheels. Nature 442:925-928.

Paxinos G, Franklin K (2001) The mouse brain in stereotaxic coordinates, Ed 2. New York: Academic.

Petersen CC (2007) The functional organization of the barrel cortex. Neuron 56:339-355.

Petersen RS, Diamond ME (2000) Spatial-temporal distribution of whisker-evoked activity in rat somatosensory cortex and the coding of stimulus location. J Neurosci 20:6135-6143.

Portera-Cailliau C, Weimer RM, De Paola V, Caroni P, Svoboda K (2005) Diverse modes of axon elaboration in the developing neocortex. PLoS Biol 3:e272.

Redecker C, Wang W, Fritschy JM, Witte OW (2002) Widespread and longlasting alterations in GABA(A)-receptor subtypes after focal cortical infarcts in rats: mediation by NMDA-dependent processes. J Cereb Blood Flow Metab 22:1463-1475.

Reinecke S, Dinse HR, Reinke H, Witte OW (2003) Induction of bilateral plasticity in sensory cortical maps by small unilateral cortical infarcts in rats. Eur J Neurosci 17:623-627.

Rossini PM, Altamura C, Ferreri F, Melgari JM, Tecchio F, Tombini M, Pasqualetti P, Vernieri F (2007) Neuroimaging experimental studies on brain plasticity in recovery from stroke. Eura Medicophys 43:241-254.

Sato TR, Gray NW, Mainen ZF, Svoboda K (2007) The functional microarchitecture of the mouse barrel cortex. PLoS Biol 5:e189.

Schaffer CB, Friedman B, Nishimura N, Schroeder LF, Tsai PS, Ebner FF, Lyden PD, Kleinfeld D (2006) Two-photon imaging of cortical surface microvessels reveals a robust redistribution in blood flow after vascular occlusion. PLoS Biol 4:e22.

Schiene K, Bruehl C, Zilles K, Qü M, Hagemann G, Kraemer M, Witte OW (1996) Neuronal hyperexcitability and reduction of GABAA-receptor expression in the surround of cerebral photothrombosis. J Cereb Blood Flow Metab 16:906-914.
Schummers J, Mariño J, Sur M (2002) Synaptic integration by V1 neurons depends on location within the orientation map. Neuron 36:969-978.

Schummers J, Mariño J, Sur M (2004) Local networks in visual cortex and their influence on neuronal responses and dynamics. J Physiol (Paris) 98:429-441.

Simons DJ (1978) Response properties of vibrissa units in rat SI somatosensory neocortex. J Neurophysiol 41:798-820.

Stosiek C, Garaschuk O, Holthoff K, Konnerth A (2003) In vivo two-photon calcium imaging of neuronal networks. Proc Natl Acad Sci USA 100:7319-7324.

Stroemer RP, Kent TA, Hulsebosch CE (1995) Neocortical neural sprouting, synaptogenesis, and behavioral recovery after neocortical infarction in rats. Stroke 26:2135-2144.

Wang X, Lou N, Xu Q, Tian GF, Peng WG, Han X, Kang J, Takano T, Nedergaard M (2006) Astrocytic Ca2+ signaling evoked by sensory stimulation in vivo. Nat Neurosci 9:816-823.

Ward NS, Brown MM, Thompson AJ, Frackowiak RS (2003a) Neural correlates of outcome after stroke: a cross-sectional fMRI study. Brain 126:1430-1448.

Ward NS, Brown MM, Thompson AJ, Frackowiak RS (2003b) Neural correlates of motor recovery after stroke: a longitudinal fMRI study. Brain 126:2476-2496.

Ward NS, Brown MM, Thompson AJ, Frackowiak RS (2006) Longitudinal changes in cerebral response to proprioceptive input in individual patients after stroke: an FMRI study. Neurorehabil Neural Repair 20:398-405.

Waters J, Helmchen F (2006) Background synaptic activity is sparse in neocortex. J Neurosci 26:8267-8277.

Watson BD, Dietrich WD, Busto R, Wachtel MS, Ginsberg MD (1985) Induction of reproducible brain infarction by photochemically initiated thrombosis. Ann Neurol 17:497-504.

Wei L, Erinjeri JP, Rovainen CM, Woolsey TA (2001) Collateral growth and angiogenesis around cortical stroke. Stroke 32:2179-2184.

Wilent WB, Contreras D (2005) Stimulus-dependent changes in spike threshold enhance feature selectivity in rat barrel cortex neurons. J Neurosci 25:2983-2991.

Winship IR, Plaa N, Murphy TH (2007) Rapid astrocyte calcium signals correlate with neuronal activity and onset of the hemodynamic response in vivo. J Neurosci 27:6268-6272.

Xerri C, Merzenich MM, Peterson BE, Jenkins W (1998) Plasticity of primary somatosensory cortex paralleling sensorimotor skill recovery from stroke in adult monkeys. J Neurophysiol 79:2119-2148.

Zepeda A, Vaca L, Arias C, Sengpiel F (2003) Reorganization of visual cortical maps after focal ischemic lesions. J Cereb Blood Flow Metab 23:811-820.

Zepeda A, Sengpiel F, Guagnelli MA, Vaca L, Arias C (2004) Functional reorganization of visual cortex maps after ischemic lesions is accompanied by changes in expression of cytoskeletal proteins and NMDA and $\mathrm{GABA}_{\mathrm{A}}$ receptor subunits. J Neurosci 24:1812-1821.

Zhang S, Murphy TH (2007) Imaging the impact of cortical microcirculation on synaptic structure and sensory-evoked hemodynamic responses in vivo. PLoS Biol 5:e119.

Zhang S, Boyd J, Delaney K, Murphy TH (2005) Rapid reversible changes in dendritic spine structure in vivo gated by the degree of ischemia. J Neurosci 25:5333-5338.

Zhu JJ, Connors BW (1999) Intrinsic firing patterns and whisker-evoked synaptic responses of neurons in the rat barrel cortex. J Neurophysiol 81:1171-1183. 